OPEN ACCESS

Edited by:

Matthew S. Francis,

Umeå University, Sweden

Reviewed by:

Hayley J. Newton,

University of Melbourne, Australia

Eric D. Cambronne,

Oregon Health and Science University,

United States

Jason King,

University of Sheffield

United Kingdom

*Correspondence:

Ludwig Eichinger

ludwig.eichinger@uni-koeln.de

Michael Steinert

steinert@tu-bs.de

Hubert Hilbi

hilbi@imm.uzh.ch

Received: 23 November 2017 Accepted: 13 February 2018

Published: 02 March 2018

Citation:

Swart AL, Harrison CF, Eichinger L,

Steinert M and Hilbi H (2018)

Acanthamoeba and Dictyostelium as

Cellular Models for Legionella

Infection.

Front. Cell. Infect. Microbiol. 8:61.

doi: 10.3389/fcimb.2018.00061

\section{Acanthamoeba and Dictyostelium as Cellular Models for Legionella Infection}

\author{
A. Leoni Swart ${ }^{1}$, Christopher F. Harrison ${ }^{2}$, Ludwig Eichinger ${ }^{3 *}$, Michael Steinert ${ }^{4 *}$ and \\ Hubert Hilbi ${ }^{1 *}$ \\ ${ }^{1}$ Institute of Medical Microbiology, Medical Faculty, University of Zurich, Zurich, Switzerland, ${ }^{2}$ Max von Pettenkofer Institute, \\ Medical Faculty, Ludwig-Maximilians University Munich, Munich, Germany, ${ }^{3}$ Institute for Biochemistry I, Medical Faculty, \\ University Hospital Cologne, Cologne, Germany, ${ }^{4}$ Department of Life Sciences, Institute of Microbiology, Technical University \\ of Braunschweig, Braunschweig, Germany
}

Environmental bacteria of the genus Legionella naturally parasitize free-living amoebae. Upon inhalation of bacteria-laden aerosols, the opportunistic pathogens grow intracellularly in alveolar macrophages and can cause a life-threatening pneumonia termed Legionnaires' disease. Intracellular replication in amoebae and macrophages takes place in a unique membrane-bound compartment, the Legionella-containing vacuole (LCV). LCV formation requires the bacterial Icm/Dot type IV secretion system, which translocates literally hundreds of "effector" proteins into host cells, where they modulate crucial cellular processes for the pathogen's benefit. The mechanism of LCV formation appears to be evolutionarily conserved, and therefore, amoebae are not only ecologically significant niches for Legionella spp., but also useful cellular models for eukaryotic phagocytes. In particular, Acanthamoeba castellanii and Dictyostelium discoideum emerged over the last years as versatile and powerful models. Using genetic, biochemical and cell biological approaches, molecular interactions between amoebae and Legionella pneumophila have recently been investigated in detail with a focus on the role of phosphoinositide lipids, small and large GTPases, autophagy components and the retromer complex, as well as on bacterial effectors targeting these host factors.

Keywords: amoebae, effector protein, GTPase, host-pathogen interaction, pathogen vacuole, phosphoinositide lipid, retrograde transport, type IV secretion

\section{LEGIONELLA SPP. -ENVIRONMENTAL BACTERIA AND OPPORTUNISTIC PATHOGENS}

Legionella spp. are the causative agents of a potentially fatal pneumonia termed Legionnaires' disease. Overall, Legionella pneumophila is the clinically most relevant species, followed by Legionella longbeachae, which is associated with outbreaks of Legionnaires' disease particularly in Australia and New Zealand (Newton et al., 2010). The Gram-negative bacteria are ubiquitously 
found in the environment, where they inhabit natural and manmade freshwater systems. Upon inhalation of Legionella-laden aerosols, the opportunistic pathogenic bacteria enter the human lung, where they infect alveolar macrophages and might cause a fulminant pneumonia (Figure 1). Legionella spp. are usually regarded to only accidentally infect humans after transmission by technical vectors such as cooling towers, fountains, or showers (Benin et al., 2002; Newton et al., 2010; Hilbi et al., 2011; Yamaguchi et al., 2017). However, recently the first probable transmission of a highly virulent L. pneumophila strain from person-to-person was reported (Correia et al., 2016).

L. pneumophila replicates intracellularly within a unique membrane compartment, which is called the Legionellacontaining vacuole (LCV). This compartment avoids acidification and fusion with lysosomes, but extensively communicates with multiple vesicle trafficking pathways, including the endosomal, secretory and retrograde route, and after a series of maturation steps associates with the endoplasmic reticulum (ER) (Isberg et al., 2009; Asrat et al., 2014; Finsel and Hilbi, 2015; Personnic et al., 2016; Bärlocher et al., 2017b). LCV formation is mechanistically similar in amoebae and macrophages and requires the $\mathrm{Icm} / \mathrm{Dot}$ (Intracellular multiplication/Defective organelle transport) type IV secretion system (T4SS) (Segal et al., 1998; Vogel et al., 1998; Segal and Shuman, 1999; Hägele et al., 2000; Solomon et al., 2000). This T4SS of L. pneumophila translocates $~ 300$ different "effector" proteins into host cells, wherein these virulence factors subvert pivotal processes such as signal transduction, cytoskeleton dynamics, and membrane trafficking (Isberg et al., 2009; Hubber and Roy, 2010; Hilbi and Haas, 2012; Haneburger and Hilbi, 2013; Sherwood and Roy, 2013; Finsel and Hilbi, 2015; Qiu and Luo, 2017). The Icm/Dot T4SS (but not the "effectorome") is conserved in the genus Legionella, including L. longbeachae, where it is also essential for virulence in amoebae and mice (Cazalet et al., 2004, 2010; Chien et al., 2004). Of note, the bioinformatics analysis of the Legionella genome sequences revealed a number of genes encoding eukaryotic-like proteins or motifs, which likely subvert host cell functions in order to allow the pathogen to survive and replicate intracellularly (Cazalet et al., 2004, 2010; Chien et al., 2004).

\section{PROTOZOA AS ENVIRONMENTAL NICHES OF LEGIONELLA SPP.}

Environmental microorganisms commonly colonize and form communities called biofilms, which comprise large numbers of prokaryotic and eukaryotic cells associating via adhesion molecules and secreted compounds. Biofilms are ubiquitously found in the environment and contain a plethora of bacterial species, many of which communicate via low molecular weight signaling molecules in a process termed quorum sensing (HallStoodley et al., 2004; Hochstrasser and Hilbi, 2017). These

Abbreviations: Icm/Dot, intracellular multiplication/defective organelle trafficking; LCV, Legionella-containing vacuole; OCRL, oculocerebrorenal syndrome of Lowe; PI, phosphoinositide; T4SS, type IV secretion system; TGN, trans-Golgi network. biofilms are attacked ("grazed") by predatory amoebae and ciliates, resulting in a reduction in the bacterial population. In contrast to most other bacterial genera, Legionella spp. resist degradation by amoebae and other protozoa (Newton et al., 2010; Hilbi et al., 2011).

In its aquatic habitats L. pneumophila replicates intracellularly in various free-living protozoa, including amoebae such as Acanthamoeba, Vermamoeba (formerly Hartmannella), Naegleria, Echinamoeba, and Vahlkampfia, as well as in ciliates like Tetrahymena (Fields, 1996; Greub and Raoult, 2004; Hilbi et al., 2011; Hsu et al., 2015). From an evolutionary point of view, interactions of L. pneumophila with protozoa shaped the relationship and likely led to adaptive responses, which enable the pathogen to also infect mammalian phagocytes such as human alveolar macrophages (Greub and Raoult, 2004; Molmeret et al., 2005; Jäger et al., 2014). In addition to their role as natural reservoir and selection niche of virulence traits, protozoa can also enhance the transmission of L. pneumophila either as intact host cell or as expelled vesicles filled with bacteria (Rowbotham, 1986; Brieland et al., 1997; Amaro et al., 2015).

A number of studies indicated that L. pneumophila is highly adapted and manages to infect a wide range of protozoan and metazoan hosts (Hilbi et al., 2011; Bergmann and Steinert, 2015; Hsu et al., 2015). This suggests that many protozoan host species and other interaction partners of L. pneumophila in the environment remain to be discovered. Recently, we reported that $L$. pneumophila, protozoa and aquatic nematodes thrive in close association within biofilms (Rasch et al., 2016). Microscopic inspection of biofilms and inoculation experiments with mCherry-labeled L. pneumophila identified the ciliates Oxytricha bifaria, Stylonychia mytilus, and Ciliophrya sp. as potential new protozoan hosts of L. pneumophila. While several metazoan organisms were identified in the biofilm (Copepoda, Nauplius larvae, Rotifera, and nematodes), only the nematodes accumulated L. pneumophila within their intestines. This correlates with the finding that the nematode Caenorhabditis elegans can be infected with L. pneumophila under laboratory conditions (Brassinga et al., 2010; Komura et al., 2010).

To study the Legionella-protozoa-nematode interactions, we infected axenically grown Acanthamoeba castellanii and $C$. elegans with $L$. pneumophila. This model system indicated that nematode larvae rupture the infected amoebae cells and thus are exposed to L. pneumophila (Rasch et al., 2016). The interaction may be different for Legionellafilled amoebae cysts and spores. D. discoideum spores resist destruction by the pharyngeal grinders of nematodes such as C. elegans and seem to be disseminated by the nematode (Kessin et al., 1996). Thus, if infected with Legionella, cysts and spores might disseminate the pathogen along this route.

L. pneumophila grown in amoebae shows distinct features compared to bacteria grown in broth (Figure 1). Accordingly, upon growth in Acanthamoeba polyphaga the bacteria are more tolerant to antibiotics (Barker et al., 1995), while grown in A. castellanii the bacteria are more virulent compared to growth on agar plates (Cirillo et al., 1994). Furthermore, the amoeba species within which replication occurs seems to also play a role, 


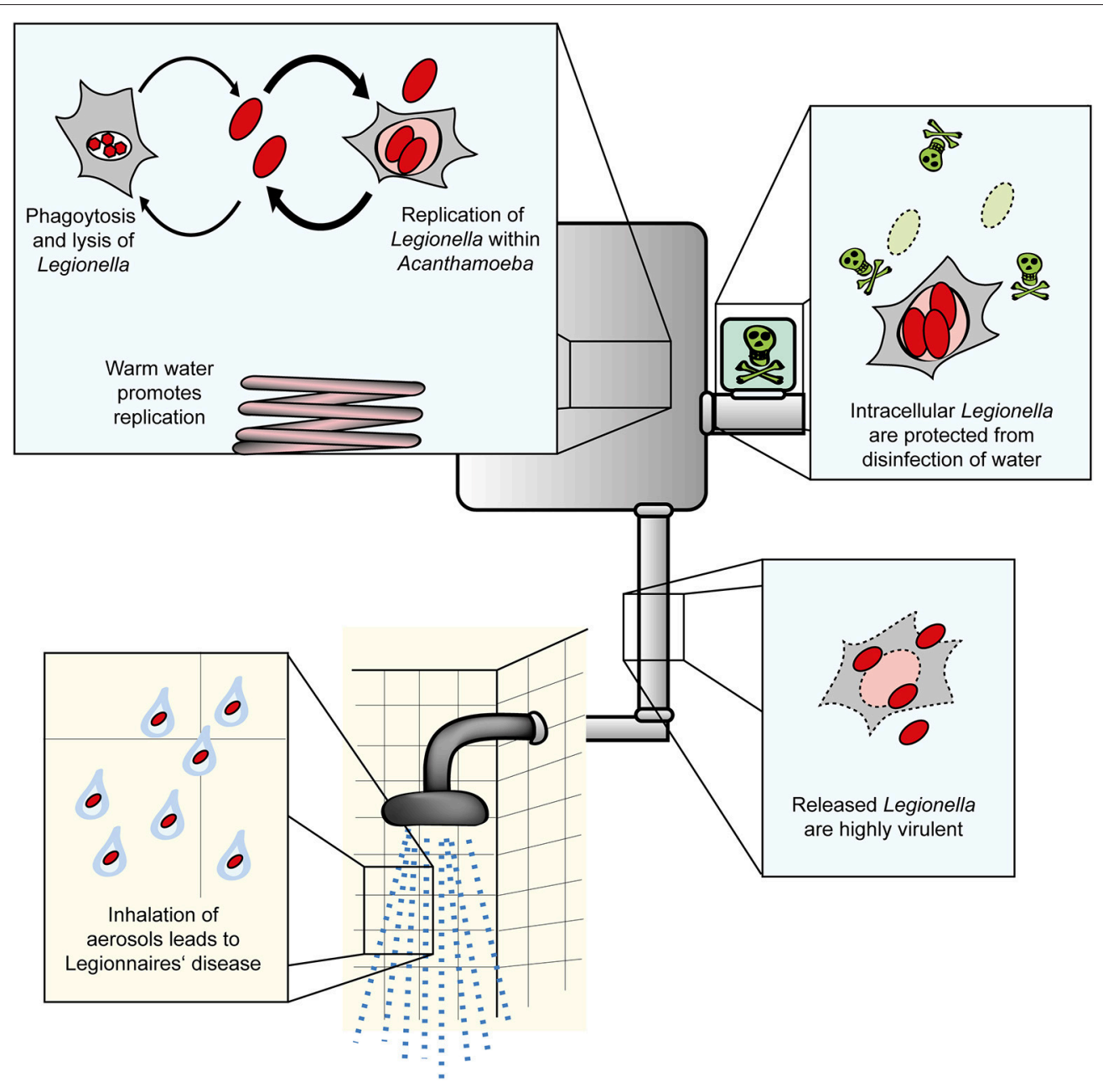

FIGURE 1 | Amoebae as natural reservoir for Legionella in water systems. L. pneumophila and amoebae are commonly isolated from natural and technical water systems. At cooler temperatures (below ca. $20-25^{\circ} \mathrm{C}$ ), L. pneumophila resists A. castellanii, but does not or only slowly replicate in the amoebae. At elevated temperatures occurring in technical water systems, L. pneumophila efficiently replicates in the amoebae. Internalized Legionella bacteria are less susceptible to routine disinfection and released from the amoebae in a highly virulent form. Virulent bacteria present in aerosols are inhaled, infect alveolar macrophages and cause Legionnaires' disease.

such that resistance to chlorination is higher in bacteria released from Hartmanella vermiformis than from A. castellanii (Chang et al., 2009).

\section{EVOLUTION AND GENE REGULATION OF LEGIONELLA IN AMOEBAE}

Legionella spp. are known to undergo horizontal gene transfer (Miyamoto et al., 2003). Gene transfer between bacteria has been shown to be significantly enhanced within the digestive vacuole of protozoa, presenting the possibility that they may act as a "trading post" to allow the acquisition of virulence genes (Schlimme et al., 1997). Perhaps reflecting this process, L. pneumophila contains a number of genes believed to be derived from eukaryotic, bacterial, or even viral sources (LurieWeinberger et al., 2010). These findings suggest that amoebae can be considered as an evolutionary niche, wherein the selective pressure and the potential for Legionella to acquire new virulence-related genes is higher than in the extracellular environment (Greub and Raoult, 2004; Molmeret et al., 2005).

An analysis of the L. pneumophila transcriptome under different growth conditions revealed that expression of almost half the genome is altered upon shifting from replicative to transmissive phase (Brüggemann et al., 2006). This was observed for bacteria grown in either broth cultures or in A. castellanii. The transmissive phase, which occurs following completion of intracellular replication, is characterized by upregulation of virulence and invasion genes, including substrates of the $\mathrm{Icm} / \mathrm{Dot}$ T4SS and motility genes (Brüggemann et al., 2006). The gene regulation of $L$. pneumophila is further complicated by the existence of non-coding (nc) RNAs, which have been shown to play a role in regulation of virulence traits (Romby et al., 2006). L. pneumophila contains a number of these regulatory ncRNAs, 
which likely are implicated in virulence, since their expression changes during the bacterial biphasic life cycle or upon infection of $A$. castellanii (Weissenmayer et al., 2011).

\section{ACANTHAMOEBA AS A NATURAL AND MODEL HOST OF LEGIONELLA}

The distinct features of the protozoan genera Acanthamoeba and Dictyostelium have been particularly useful in examining the ecology and cellular host-interactions of L. pneumophila. Given the similarity of the infection process in amoebae and macrophages, the amoebae are powerful models to study bacteria-macrophage interactions (Hilbi et al., 2007; Escoll et al., 2013; Hoffmann et al., 2013; Bergmann and Steinert, 2015). Acanthamoeba has often been found in Legionella-positive habitats and has an extremely wide distribution. The amoebae have been isolated from diverse aquatic environments, including soil (Sawyer, 1989), roadside puddles (Sakamoto et al., 2009), fresh water lakes and rivers (John and Howard, 1995), frozen lakes (Brown and Cursons, 1977), the atmosphere (RodriguezZaragoza et al., 1993), and even the Antarctic (Brown et al., 1982).

The majority of Acanthamoeba isolates from these aquatic environments harbor endosymbionts, such as viruses, bacteria, yeast, and protists (Greub and Raoult, 2004). However, for laboratory studies of host-pathogen interactions Acanthamoeba strains adapted to axenic growth are mostly used (Shevchuk et al., 2014; Eisenreich and Heuner, 2016). Like many other free-living amoebae, Acanthamoeba adopts a bi-phasic life cycle, comprising a vegetative trophozoite stage and a dormant cyst stage, which at least in part might explain the wide environmental range of these protozoa (Chávez-Munguía et al., 2005). L. pneumophila utilizes the trophozoites for replication and the double-walled cysts as shelter to escape harsh environmental conditions (Fields, 1996).

Acanthamoeba replicates by binary fission and, under culturing conditions, has a doubling time reported to range from 8 to $18 \mathrm{~h}$ (Berk and Garduño, 2013). The amoebae are highly motile and follow a random walk pattern with a movement speed of $0.1-0.2 \mu \mathrm{m} / \mathrm{s}$. In the presence of chemical signals produced by bacteria, Acanthamoeba will use chemotaxis to "hunt," actively moving toward the source of these signals (Preston and King, 1984; Schuster and Levandowsky, 1996). Moreover, Acanthamoeba spp. are characterized by contractile vacuoles, which expel water for osmotic regulation (Bowers and Korn, 1973), and they contain glycogen storage vacuoles, lysosomes and digestive vacuoles, wherein L. pneumophila seems to replicate (Ulsamer et al., 1971; Bowers and Korn, 1973; Eisenreich and Heuner, 2016). Actin microfilaments adjacent to the plasma membrane of $A$. castellanii are responsible for forming protrusions (Pollard et al., 1970), and trophozoites produce short, spine-like projections at the edge of the cell, known as acanthapodia. As the amoebae are very motile, these projections are short-lived but constantly replaced, normally collapsing after less than a minute during active movement (Preston and King, 1984). A. castellanii also employs a diverse repertoire of putative pattern recognition receptors (PRRs), many of which with postulated orthologous functions in the innate immune systems of higher organisms (Clarke et al., 2013).

The genome of $A$. castellanii is polyploid and harbors $\sim 15,500$ compact intron-rich genes, a number of which are predicted to have been acquired through inter-kingdom lateral gene transfer (Clarke et al., 2013). The genomic complexity of A. castellanii remains one of the barriers to its broad utilization as a model organism. Transfection has been predominantly unsuccessful, with only one report of success (Peng et al., 2005). Furthermore, the polyploid genome organization prevents simple knockout of genes of interest, although approaches using RNA interference to knock down gene expression have been successfully used (Lorenzo-Morales et al., 2005). Overall, however, the known sequence of the A. castellanii genome and the increasing number of available molecular tools will strongly augment ecological and cellular research with this authentic host of L. pneumophila.

\section{DICTYOSTELIUM DISCOIDEUM: A VERSATILE CELLULAR MODEL FOR LEGIONELLA INFECTION}

In contrast to Acanthamoeba spp., Dictyostelium discoideum is not a prevalent natural host for L. pneumophila in the environment. This social amoeba, which naturally lives in forest soil and has a narrow temperature tolerance of 20$25^{\circ} \mathrm{C}$, undergoes a complex developmental program from a single cell to become a multicellular organism (Taylor et al., 2009; Bretschneider et al., 2016). D. discoideum replicates by mitotic division of single amoebae. These amoeba cells live of bacteria, which are taken up by phagocytosis. Laboratory strains, e.g., Ax2, can also thrive in liquid medium through macropinocytosis. Exhaustion of the nutrient supply triggers an intricate multicellular cooperativity. In response to cAMP signals, solitary cells aggregate by chemotaxis, and $\sim 100,000$ cells form a migrating slug, which responds thermo-tactically and photo-tactically. At the final stage of morphogenesis a fruiting body is formed, consisting of a basal disc, a stalk, and spores. Accordingly, the genome of $D$. discoideum encodes morphogenetic traits such as cell-type determination, spatial patterning, cell death, and other features that are essential in multicellular but not unicellular organisms (Steinert and Heuner, 2005; Steinert, 2011; Schilde et al., 2016). The genome of D. discoideum is about $34 \mathrm{Mb}$ and comprises six chromosomes, which range from 3.5 to $8.6 \mathrm{Mb}$ in size. Most genes contain introns with conserved splice junctions. About 100 times smaller than the human genome, the $D$. discoideum genome encodes $\sim 12,500$ predicted proteins, including a large number of mammalian orthologs (Eichinger et al., 2005). In addition, D. discoideum is increasingly used as a model for the study of genes that in mutant form cause disease in humans (MüllerTaubenberger et al., 2013).

The completed genome sequence of $D$. discoideum, a remarkable repertoire of molecular genetic tools, and the intrinsic biological features of the amoebae allow to study many fundamental cellular processes (Bergmann and Steinert, 2015; Bretschneider et al., 2016; Hochstrasser and Hilbi, 
2017). In the haploid amoeba $D$. discoideum non-essential genes can be easily disrupted or replaced by homologous recombination. Further genetic manipulation strategies include random insertion mutagenesis (restriction-enzyme-mediated integration, REMI), multiple gene deletions, Cre/LoxP-mediated recombination, RNA interference techniques, and ectopic expression of endogenous or foreign genes (Kuspa and Loomis, 1992; Chen et al., 1994; Faix et al., 2004; Kuhlmann et al., 2006; Al-Quadan and Kwaik, 2011). The large mutant collection of the Dictyostelium stock center (http://www.dictybase.org) (Gaudet et al., 2011), easy cultivation, amenability to diverse biochemical and biological approaches, such as in vivo expression of fluorescence-tagged proteins, also contributed to the present strength and versatility of the $D$. discoideum model. Recent highlights resulting from using this model host of L. pneumophila will be discussed below.

\section{INTRACELLULAR REPLICATION AND COMPETITION OF L. PNEUMOPHILA IN AMOEBAE}

The amoeba plate test is a relatively simple assay to determine the ability of L. pneumophila mutants to form colonies in the presence of A. castellanii (Albers et al., 2005) (Figure 2A). A suspension of $A$. castellanii or media alone is spread onto charcoal yeast extract (CYE) agar plates, and allowed to dry. Serial dilutions of $L$. pneumophila are spotted onto the plates and incubated at 30 or $37^{\circ} \mathrm{C}$ for 3 days. This method allows comparing the growth of multiple strains on a single plate, in both the presence and absence of amoebae. In this manner, L. pneumophila mutants defective for amoebae resistance and intracellular replication can be determined in a semi-quantitative manner, alongside bacterial strains capable of suppressing these phenotypes (Albers et al., 2005). A variant of this method has been employed to screen clonal libraries of $>20,000$ L. pneumophila mutants for virulence defects (Aurass et al., 2009). In this approach, mutagenized bacteria are used to infect A. castellanii prior to plating.

While the amoeba plate test allows screening of strong replication phenotypes, the resulting output is not easily quantified. A more detailed analysis can be performed by measuring the number of bacteria present within or released by amoebae at given time points by determining bacterial colony forming units (CFU). A. castellanii or D. discoideum is infected with L. pneumophila in Ac medium or MB medium, respectively. In these media L. pneumophila is unable to replicate, which allows detection of intracellular replication exclusively. The host cells are then lysed by shear forces or mild detergent treatment, followed by plating on CYE agar to determine CFU. This assay has been used to assess the infection and replication of L. pneumophila, L. dumoffii, and L. feelii in A. castellanii (Moffat and Tompkins, 1992). Upon uptake of L. pneumophila, bacterial CFU drop significantly in the first few hours. Thereafter, the pathogen replicates intracellularly, leading to a 100-1,000 fold increase in bacterial numbers, and at the end of the cycle, the bacteria escape and disseminate. Bacterial amoebae resistance and intracellular replication can also be applied to screening of water samples. In this approach, samples negative for Legionella by normal culture techniques are incubated with amoebae, allowing intracellular growth of the bacteria to easily detectable numbers (Sanden et al., 1992).

Faster and more sensitive methods to determine intracellular replication of L. pneumophila in amoebae utilize an automated microtiter plate reader. This approach allows to measure the increase in optical density at $600 \mathrm{~nm}$ (Coil et al., 2008) or bacteria-produced fluorescence, e.g., GFP (Harrison et al., 2013, 2015a,b) (Figure 2B). To monitor intracellular growth by fluorescence increase over time, A. castellanii or $D$. discoideum is infected at $30 / 37^{\circ} \mathrm{C}$ or maximum at $25^{\circ} \mathrm{C}$, respectively, with either GFP- or mCherry-producing L. pneumophila strains. To this end, we preferentially use LoFlo medium, a commercially available, complex $D$. discoideum medium that exhibits low auto-fluorescence and does not allow extracellular growth of L. pneumophila. The fluorescent signal for each sample is measured by microtiter plate reader at various time points post infection (p.i.). Since the fluorescence intensity is proportional to the number of bacteria present in a sample, the quantification of fluorescence increase reflects the rate of intracellular bacterial replication. Using the CFU or fluorescence assays, a number of L. pneumophila regulators or effector proteins, including LqsR (Tiaden et al., 2007), RidL (Finsel et al., 2013b), LppA (Weber et al., 2014a), and LegG1 (Rothmeier et al., 2013), have been found to promote intracellular replication in amoebae.

In contrast, the deletion of some other regulators (LqsA), effectors (SidC, SidM), or unknown proteins (HdeD) did not result in an intracellular growth defect of L. pneumophila. This might be due to robust intracellular replication of L. pneumophila involving redundant effectors, or simply because a gene is not required under the conditions tested. To discover weaker phenotypes of L. pneumophila mutant strains, we established the more sensitive amoebae competition test (Kessler et al., 2013). In this assay, A. castellanii is co-infected with the L. pneumophila parental and deletion mutant strain at a 1:1 ratio (multiplicity of infection of 0.01 each) and grown at $37^{\circ} \mathrm{C}$ for $15-21$ days. Every third day the supernatant and lysed amoebae are diluted 1:1000, fresh amoebae are infected, and CFU are determined on agar plates containing kanamycin (to select for presence of the resistance cassette in the mutant strains) or not. Using this approach, L. pneumophila mutant strains lacking individual Lqs components (Kessler et al., 2013) or single effectors (LegG1, RidL, LppA, SidC) were outcompeted by the parental strain (Finsel et al., 2013b; Rothmeier et al., 2013; Dolinsky et al., 2014; Weber et al., 2014a). Thus, in the course of successive rounds of infection the fitter strain (usually the parental strain) will come to dominate the bacterial pool.

Using these assays, in addition to L. pneumophila mutant strains, the effect of host proteins on intracellular bacterial replication can also be assessed. Upon pharmacological inhibition of phosphoinositide (PI) 3-kinases (PI3Ks) by wortmannin or LY294002, or deletion of the kinases in D. discoideum, L. pneumophila replicated more efficiently, and therefore the PI3Ks restrict intracellular growth (Weber et al., 2006). In contrast, overproduction of the atlastin (Atl) homolog 


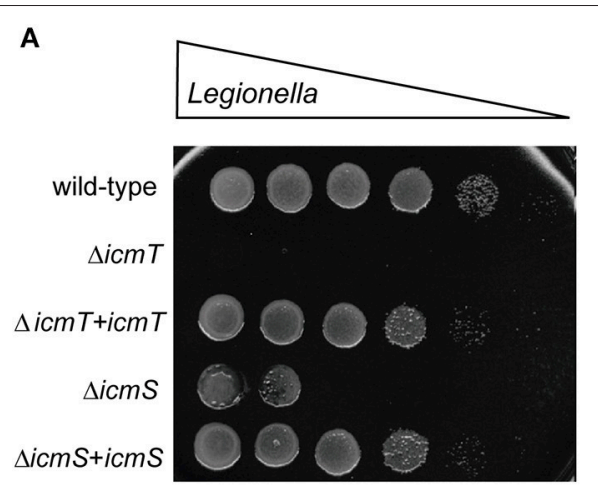

C

brightfield

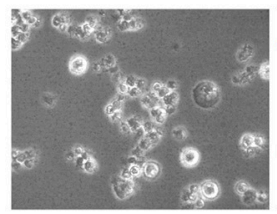

E
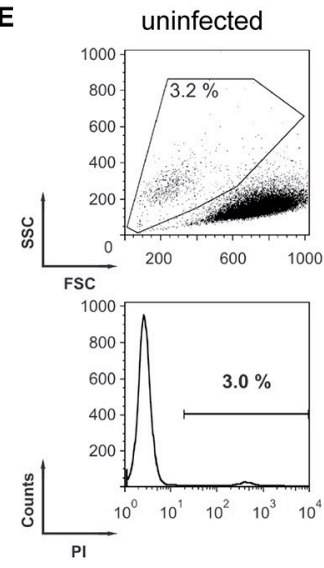

propidium lodide
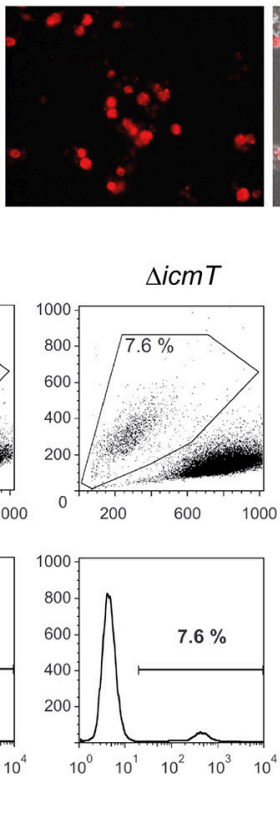

G
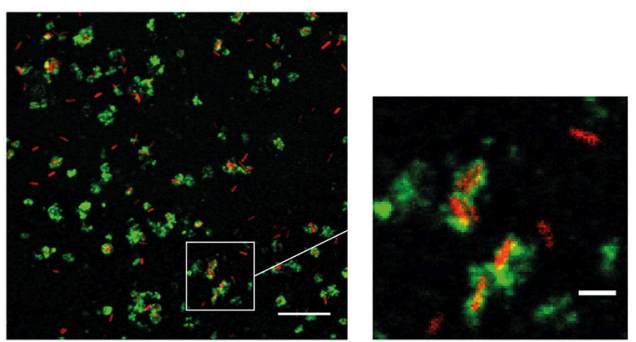
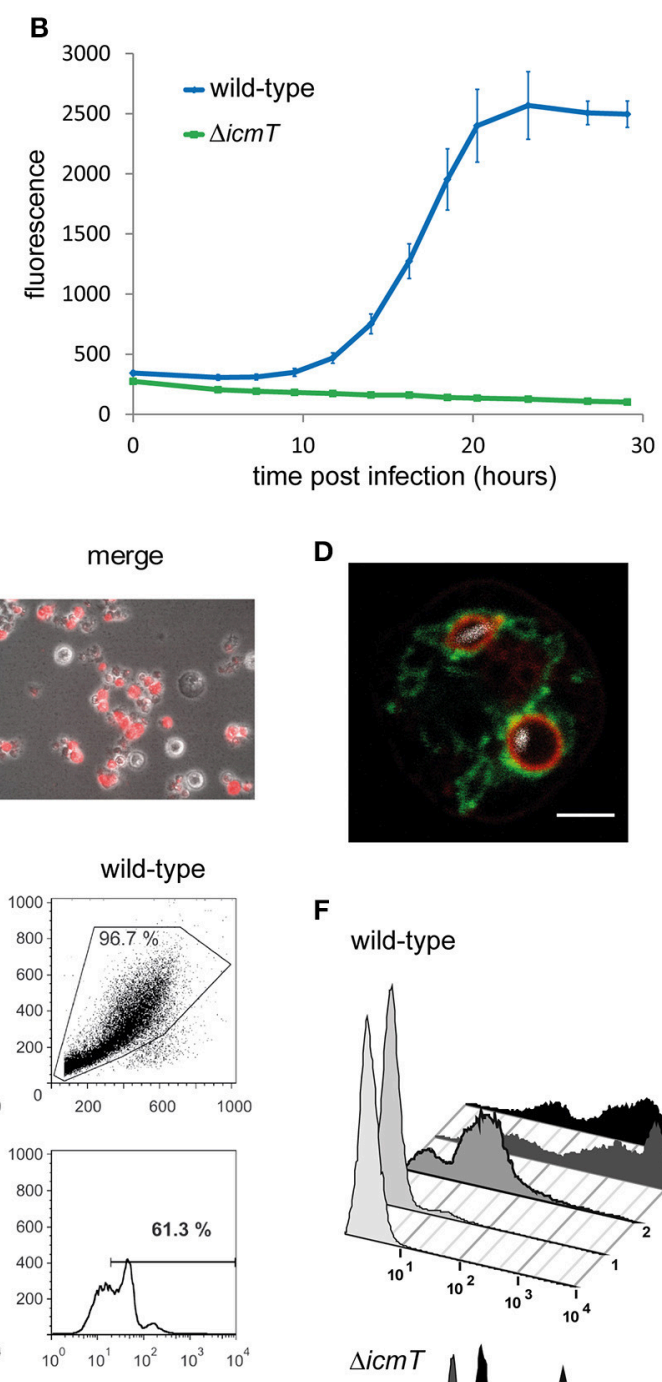

merge
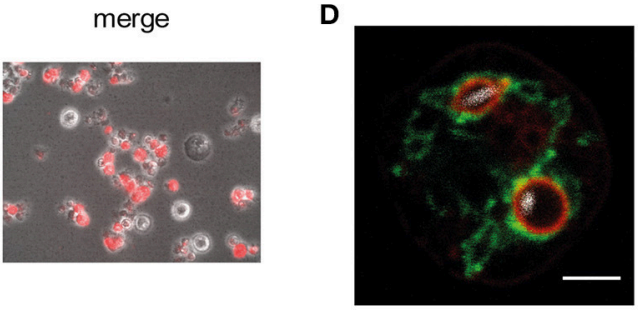

F
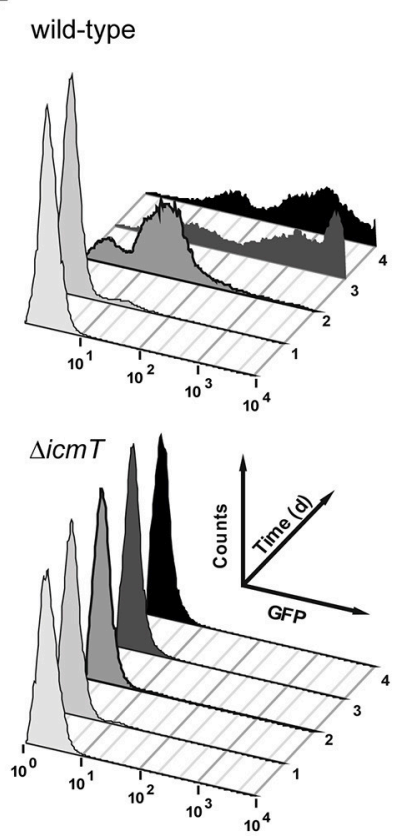

FIGURE 2 | Experimental approaches to study Legionella infection of amoebae. (A) Amoeba plate test showing serial dilutions of bacterial cultures spotted on a lawn of $A$. castellanii. Virulent $L$. pneumophila is amoeba-resistant and grows in high dilutions, while mutant strains lacking a functional Icm/Dot T4SS do not ( $\Delta i c m T)$ or barely $(\Delta i \mathrm{cmS})$ grow. Growth defect of the mutants is complemented by plasmid-borne expression of the corresponding genes $(\Delta i \mathrm{~cm} T+i \mathrm{~cm} T, \Delta i \mathrm{~cm} S+i \mathrm{~cm} S)$.

(B) Intracellular replication quantified by fluorescence from GFP-producing $L$. pneumophila. Virulent bacteria show an increase in fluorescence over time, while $\Delta i c m T$ mutant bacteria do not grow. (C) Fluorescence microscopy images of $L$. pneumophila-infected, dying amoebae that round up, losing the characteristic spiky morphology, and take up the fluorescent dye propidium iodide. (D) Live-cell confocal fluorescence microscopy of $D$. discoideum strain Ax3 producing P4C-mCherry (red) and GFP-Sey1 (green), infected (MOI 10, 2 h) with mCerulean-producing L. pneumophila JR32 (white). Scale bar: $2 \mu \mathrm{m}$. (E) Flow cytometry of A. castellanii infected with $L$. pneumophila wild-type or $\Delta i c m T$ and stained with propidium iodide. Dye uptake and changes in morphology are quantified by fluorescence and light scattering, respectively. (F) Flow cytometry gating on infected $A$. castellanii reveals replication of GFP-producing intracellular $L$. pneumophila wild-type but not $\triangle i c m T$ mutant bacteria. (G) LCV isolation by immuno-magnetic separation and density gradient centrifugation from lysates of $D$. discoideum Ax3, infected (MOI 10, $2 \mathrm{~h}$ ) with DsRed-producing L. pneumophila JR32 (red). The preparation was immuno-stained with an anti-calnexin antibody, followed by a FITC-coupled secondary antibody (green). Scale bar: $10 \mu \mathrm{m}$, zoom: $2 \mu \mathrm{m}$. Image (A) was reproduced with permission from Albers et al. (2005) and images (C,E,F) from Tiaden et al. (2007). 
Sey1 in D. discoideum promoted intracellular growth, while production of the catalytically inactive, dominant negative mutant protein Sey1_K154A or depletion by RNAi of Atl3 in mammalian cells inhibited intracellular replication of L. pneumophila (Steiner et al., 2017a).

\section{THE TRANSCRIPTIONAL RESPONSE OF $D$. DISCOIDEUM UPON INFECTION WITH L. PNEUMOPHILA}

In several infection models, including $D$. discoideum and human macrophages, it has been shown that the quantitative and qualitative modulation of diverse host cell functions is crucial for efficient intracellular replication of L. pneumophila (Bozzaro and Eichinger, 2011; Hochstrasser and Hilbi, 2017). Accordingly, the analysis of the transcriptional changes that occur in $D$. discoideum upon infection with L. pneumophila revealed important aspects of the complex host-pathogen crosstalk (Farbrother et al., 2006). The experiment was performed with cDNA-microarrays, covering approximately half of the Dictyostelium genome, and led to the identification of 731 differentially regulated host genes in a $48 \mathrm{~h}$ infection with the virulent $L$. pneumophila Philadelphia-1 strain JR32. In addition, a detailed analysis of the $24 \mathrm{~h}$ time point p.i. of L. pneumophila JR32, an avirulent L. pneumophila $\Delta \operatorname{dotA}$ mutant, or Legionella hackeliae (reduced virulence), uncovered 131 differentially expressed genes common to all three strains compared with uninfected amoebae. The corresponding gene products are either involved in host-specific defense mechanisms or required for successful infection and proliferation of L. pneumophila (Farbrother et al., 2006).

Functional annotation of the differentially regulated genes of the $48 \mathrm{~h}$ and $24 \mathrm{~h}$ time points revealed that in addition to triggering a stress response, L. pneumophila not only interferes with intracellular vesicle trafficking, but also subverts and exploits the host metabolism. A considerable enrichment of differentially regulated genes involved in translation, proteolysis, nucleotide metabolism, and lipid modification was observed. Moreover, L. pneumophila induces the expression of multiple amino-acyl t-RNA synthetases (aaRSs). Interestingly, besides the canonical coupling of correct amino acids to t-RNA molecules, aaRSs were described to play an unexpected role in splicing, apoptosis, and regulation of transcription and translation (Hausmann and Ibba, 2008; Yannay-Cohen et al., 2009). Thus, the upregulation of $D$. discoideum aaRSs upon L. pneumophila infection may serve a similar function in different signaling pathways. The transcriptome results also showed that infection with $L$. pneumophila results in differential expression of host genes that are involved in bacterial degradation and autophagy. Additionally, genes encoding cytoskeleton proteins were enriched $\sim 7$-fold during the early stage of infection. These findings likely reflect the uptake of bacteria and transport of the nascent phagosome inside the host cell (Farbrother et al., 2006).

In a different study it was found that infection of D. discoideum by L. pneumophila caused a decrease in mitochondrial messenger RNAs and a cleavage of the mitochondrial large subunit ribosomal RNA (LSU rRNA). The specific cleavage of the LSU rRNA in D. discoideum required functional L. pneumophila type II and type IV secretion systems. LSU rRNA cleavage was, however, not observed upon infection of A. castellanii or human U937 macrophages, suggesting that L. pneumophila might use distinct mechanisms to interrupt the mitochondrial metabolism in different hosts (Zhang and Kuspa, 2009).

In summary, the transcriptional changes in L. pneumophilainfected $D$. discoideum suggest complex and still poorly understood regulatory interactions between pathogen and host. In the course of establishing its replicative niche, L. pneumophila not only interferes with signaling processes, mitochondrial function, and intracellular vesicle trafficking, but also profoundly influences the metabolism of its host.

\section{ANALYSIS OF LEGIONELLA INFECTION IN DEFINED D. DISCOIDEUM MUTANT STRAINS}

Investigations of $D$. discoideum mutant strains have significantly contributed to our understanding of Legionella infection. Host cell factors implicated in host-pathogen interactions are either involved in the cellular defense or exploited by the pathogen in the course of infection. Consequently, the inactivation of distinct host factors may either favor or impede Legionella infection. To identify host genes crucial for Legionella infection, candidate gene approaches as well as untargeted mutational screens have been carried out in D. discoideum (Bozzaro et al., 2008; Li et al., 2009). In the candidate gene approach, either available $D$. discoideum mutants are used, which in many cases can be ordered from the Dictyostelium stock center (Fey et al., 2013), or novel genes of interest are disrupted, silenced, or the corresponding proteins overproduced as fluorescently tagged fusions (Bozzaro et al., 2008; Steinert, 2011; Steiner et al., 2017b). In order to dissect the complex cross-talk between $D$. discoideum and L. pneumophila, classical infection assays were performed, and a versatile methodology has been developed based on fluorescently tagged marker proteins, heterologously produced bacterial effectors and different visualization techniques (Eichinger and Rivero, 2006; Steinert, 2011; Steiner et al., 2017b). As select examples, we will discuss the role of the host cytoskeleton, autophagy machinery, and phospholipid metabolism in the infection process.

Most D. discoideum knock-out mutants of cytoskeletal proteins caused a decrease in the uptake and in the proliferation of L. pneumophila, indicating that an intact cytoskeleton is important (Bozzaro et al., 2013). Exceptions to this rule were the coronin A and B genes. Coronins comprise a large family of proteins that function mainly in actin cytoskeletonassociated processes. The proteins harbor a WD (Trp-Asp)repeat domain containing seven repeats that form a sevenbladed $\beta$-propeller, adjacent to a unique region, and a $\mathrm{C}$ terminal coiled coil region which mediates trimerization (Shina and Noegel, 2008). We studied uptake and replication of L. pneumophila in D. discoideum mutants where corA (encoding the conventional coronin), $\operatorname{cor} B$ (encoding coronin 7 (CRN7)), or 
vilA (encoding villidin a member of the coronin 4 family) were disrupted. Absence of conventional coronin reduced the uptake of L. pneumophila and enhanced intracellular growth. Villidindeficient cells showed an even higher reduction in uptake, and intracellular growth was strongly reduced (Fajardo et al., 2004). In contrast, uptake of L. pneumophila was enhanced in CRN7deficient cells but intracellular replication was not affected. Overexpression of CRN7 on the other hand caused reduced internalization and an increased replication of L. pneumophila, suggesting that CRN7 negatively regulates the internalization of the bacteria. A double mutant wherein the $\operatorname{cor} A$ and $\operatorname{cor} B$ genes were inactivated behaved like the corA deletion strain (Shina et al., 2010, 2011) (Table 1). CRN7 harbors a Cdc42- and Racinteractive binding (CRIB) domain in each of its two $\beta$-propeller domains with a preference for GDP-loaded Rac. Thus, CRN7 might keep Rac GTPases in their GDP-bound form and locally prevent the activation of the downstream targets SCAR and WASP. Loss of CRN7 hyper-activates WASP, which promotes Factin assembly leading to increased phagocytosis (Swaminathan et al., 2015).

In recent years, autophagy has been identified as an ancient and important cellular defense mechanism that targets intracellular pathogens for destruction (Sherwood and Roy, 2016). A number of pathogens are able to subvert this cellular defense, and also L. pneumophila interferes with the autophagy process of the host. In early studies with $D$. discoideum mutants deficient for either one of five different core autophagy genes autophagy appeared to be dispensable for intracellular replication of L. pneumophila at later stages of infection (Otto et al., 2004). However, infection assays with atg9, atg16, and atg9/16 deletion mutants revealed a reduced uptake of $L$. pneumophila in these strains in comparison to Ax2 wild-type cells (Tung et al., 2010; Xiong et al., 2015). For the atg9 deletion mutant it was further shown that those bacteria that entered the host were initially cleared less efficiently and at later stages of the infection multiplied more efficiently compared to the parental amoebae (Tung et al., 2010) (Table 1). It must be kept in mind that apart from its role in autophagy ATG9 likely has additional roles in trafficking, as it is localized to a number of cytoplasmic vesicular structures (Noda, 2017). Noteworthy, atg9 and atg16 were oppositely regulated in parental $D$. discoideum in response to infection (Farbrother et al., 2006; Tung et al., 2010).

The L. pneumophila Icm/Dot substrate RavZ is a cysteine protease that irreversibly cleaves lipid-conjugated Atg8 proteins on the membranes of nascent autophagic structures (Choy et al., 2012). Furthermore, the L. pneumophila sphingosine-1 phosphate lyase (LpSpl) targets host sphingolipid metabolism and inhibits autophagy in mouse macrophages (Rolando et al., 2016). These findings suggest that both, the host cell and the pathogen, modulate autophagy for their own benefit.

Another cellular process that is part of the defense reaction of the host and exploited by L. pneumophila is the PI lipid metabolism (Weber et al., 2009b; Haneburger and Hilbi, 2013). PI lipids are important for vesicle trafficking and organelle identity, since the cellular compartments are defined in part by their PI composition. The lipid head group can be phosphorylated or dephosphorylated at the $3^{\prime}, 4^{\prime}$, and/or $5^{\prime}$ positions by specific PI kinases and PI phosphatases, resulting in an array of seven specific PIs (Di Paolo and De Camilli, 2006). Deletion or pharmacological inhibition of PI3Ks class I promotes intracellular replication of L. pneumophila and impairs the transition from tight to spacious LCVs (Weber et al., 2006). Disruption of $D$. discoideum PTEN (phosphatase and tensin homolog), a PI phosphatase antagonizing PI3Ks, reduces the uptake but does not affect proliferation of L. pneumophila (Peracino et al., 2010). D. discoideum PLC (phospholipase C) is involved in the PI metabolism through hydrolysis of $\mathrm{PI}(4,5) \mathrm{P}_{2}$ to diacylglycerol (DAG) and inositol 1,4,5-triphosphate $\left(\mathrm{IP}_{3}\right)$. Its inhibition dramatically reduced the engulfment of L. pneumophila, but had no effect on bacterial replication (Peracino et al., 2010).

Furthermore, in D. discoideum cells lacking the PI 5-phosphatase Dd5P4, a homolog of human OCRL (oculocerebrorenal syndrome of Lowe), bacterial replication and LCV formation occurred more efficiently. Dd5P4 localizes to LCVs via its $\mathrm{N}$-terminus and might interact with the L. pneumophila PI(3)P-binding virulence factor LpnE (Weber et al., 2009a). The PI 5-phosphatase is catalytically active on the LCV, thereby increasing the PI(4)P available for binding of the L. pneumophila effector proteins SidC or SidM. An additional host protein, which plays a role in the PI metabolism is RpkA, an unusual seven-helix trans-membrane receptor with a predicted intracellular PI(4)P 5-kinase activity. RpkA is specific for lower eukaryotes and is recruited to nascent LCVs in D. discoideum. It disturbs the PI balance and thereby plays a role in the defense against L. pneumophila. RpkA interacts with the V-ATPase complex, but whether it can actively recruit the V-ATPase to the LCV is not known (Riyahi et al., 2011) (Table 1).

These examples illustrate the power of the D. discoideum system for the investigation of the host side during infection. The biological properties of this amoeba in combination with the straight-forward generation and analysis of mutant strains will continue to provide a potent and versatile model for the dissection of the complex cross-talk with L. pneumophila.

\section{FLUORESCENCE MICROSCOPY OF LEGIONELLA-INFECTED LABELED D. DISCOIDEUM}

The difficulty of performing genetic manipulations in A. castellanii implies that modifications of host cell factors, for example the production of GFP-labeled proteins, are not feasible. One of the few fluorescence assays that can be performed is the determination of Legionella-triggered cytotoxicity. To this end, the number of infected $A$. castellanii cells permeable to fluorescent dyes such as propidium iodide is counted, as has been done to assess the cytotoxicity of L. pneumophila deletion mutants (Albers et al., 2007) (Figure 2C). Given these constraints, genetically tractable amoebae such as D. discoideum, are more useful for cell biology and pathogen-host interaction studies, which examine the localization of specific markers to the LCV.

A plethora of genetic tools is available to study hostpathogen interactions using $D$. discoideum. In addition to DNA microarrays and targeted deletions or random mutations of 
TABLE 1 | Selected D. discoideum mutants implicated in L. pneumophila interactions.

\begin{tabular}{|c|c|c|c|c|}
\hline \multirow[t]{2}{*}{ Host cell factor } & \multirow[t]{2}{*}{ Manipulation } & \multicolumn{2}{|c|}{ Effects on infection: } & \multirow[t]{2}{*}{ References } \\
\hline & & Uptake & Growth & \\
\hline ATG 9 & Knockout & Down & Up & Tung et al., 2010 \\
\hline ATG 16 & Knockout & Down & n.t. & Xiong et al., 2015 \\
\hline ATG 9/ATG 16 & Double knockout & Down & n.t. & Xiong et al., 2015 \\
\hline \multirow[t]{2}{*}{ Coronin } & Knockout & Down & Up & Solomon et al., 2000; Fajardo et al., 2004 \\
\hline & & n.t. & Up & \\
\hline \multirow[t]{2}{*}{ Coronin 7} & Knockout & Up & Normal & Shina et al., 2010 \\
\hline & Overexpression & Down & Normal & \\
\hline Coronin/coronin 7 & Double knockout & Down & Up & Shina et al., 2011 \\
\hline Dd5P4 (OCRL) & Knockout & Down & Up & Weber et al., 2009a \\
\hline PLC & Inhibitors & Down & Normal & Peracino et al., 2010 \\
\hline $\mathrm{PI3K} 1 / 2$ & Double knockout & Normal & Up & Weber et al., 2006 \\
\hline $\mathrm{PI3K}$ 1/2/3/4/5/6 & Sextuple knockout & Down & Up & Peracino et al., 2010 \\
\hline PI3K 1/2/3/4/5/6/PTEN & Septuple knockout & Down & Up & Peracino et al., 2010 \\
\hline PTEN & Knockout & Down & Normal & Peracino et al., 2010 \\
\hline RpkA & Knockout & Normal & Up & Riyahi et al., 2011 \\
\hline \multirow[t]{2}{*}{ Sey1 } & Overexpression (WT) & n.t. & Up & Steiner et al., 2017a \\
\hline & Overexpression (DN) & n.t. & Down & \\
\hline TBC1D5 & Knockout & n.t. & Down & Bärlocher et al., 2017a \\
\hline Villidin & Knockout & Down & Down & Fajardo et al., 2004 \\
\hline
\end{tabular}

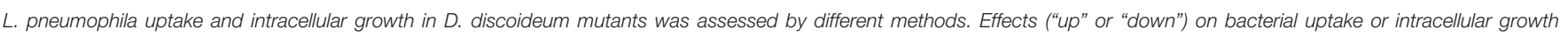

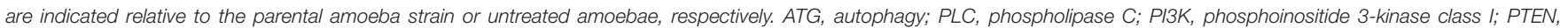

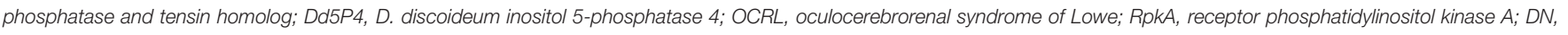
dominant negative; WT, wild-type; n.t.: not tested.

genes, an extensive library of plasmids is available allowing constitutive or inducible production of fluorescently labeled probes in D. discoideum (http://wiki.dictybase.org/dictywiki/ index.php/Vectors). Specifically, various expression vectors for $\mathrm{N}$ - or C-terminal fusions with green- or red-fluorescent proteins are available. These include extrachromosomal (Levi et al., 2000), extrachromosomal inducible expression (Veltman and van Haastert, 2013), or integrating plasmids (Manstein et al., 1995), as well as vectors for efficient generation of gene knockouts using one-step cloning (Wiegand et al., 2011). For the production of fluorescent probes, $D$. discoideum is transformed with one or multiple plasmids by electroporation (Weber et al., 2014b). Transformants are isolated by antibiotic selection and form microcolonies within 7-12 days after transformation.

A GFP-fusion protein commonly used in D. discoideum is calnexin-GFP, an ER-specific type I transmembrane protein and well-established LCV marker (Fajardo et al., 2004; Weber et al., 2006; Ragaz et al., 2008; Dolinsky et al., 2014). Within $2 \mathrm{~h}$ after uptake, calnexin accumulates on the membrane of LCVs containing virulent $L$. pneumophila, but not avirulent $\Delta i c m T$ mutant bacteria, and to a significantly smaller extent on vacuoles harboring $\Delta$ sidC mutants (Ragaz et al., 2008; Dolinsky et al., 2014; Weber et al., 2014b). Using D. discoideum cells producing fluorescently labeled proteins, numerous other host proteins were found to localize to the LCV. These include small GTPases of the Rab family (Urwyler et al., 2009; Hoffmann et al., 2014), Rap1 (Schmölders et al., 2017), and Ran (Rothmeier et al., 2013), the ER tubule-resident large GTPase Atl3/Sey1 (Steiner et al., 2017a) (Figure 2D), as well as components implicated in the retrograde vesicle trafficking pathway, such as the $D$. discoideum OCRL homolog Dd5P4 (Weber et al., 2009a) and subunits of the retromer complex (Finsel et al., 2013a).

A caveat of microscopy methods using fixed samples is that the fixation techniques might alter the morphology of cellular structures such as membranes, vesicles, or microtubules. Timelapse imaging of living cells on the other hand is optimal to follow cellular dynamics over time. A well-studied process is the PI conversion on the LCV membrane during infection (Weber and Hilbi, 2014; Weber et al., 2014b). L. pneumophilainfected $D$. discoideum producing various PI probes, including $\mathrm{PH}_{\text {CRAC-GFP }}$ for $\mathrm{PI}(3,4,5) P_{3}$ and $\mathrm{PI}(3,4) P_{2}, 2 \times$ FYVE-GFP for $\mathrm{PI}(3) P$ or GFP-P4C $\mathrm{C}_{\text {SidC }}$ for $\mathrm{PI}(4) P$, were imaged live for time-lapse analysis (Weber et al., 2014b). Immediately upon bacterial uptake, the phagosome containing L. pneumophila transiently accumulates $\mathrm{PI}(3,4,5) P_{3}$ for up to $1 \mathrm{~min}$, and within that time-span, $\mathrm{PI}(3) P$ is acquired in a $\mathrm{Icm} / \mathrm{Dot}$ independent manner. During the following $2 \mathrm{~h}$, LCVs containing virulent L. pneumophila gradually replace $\mathrm{PI}(3) P$ with $\mathrm{PI}(4) P$, whereas $\Delta i c m T$ vacuoles remain PI(3)P positive (Weber et al., 2014b). Using dually labeled $D$. discoideum, the P4C-mRFPmars signal was spatially separated from calnexin-GFP-positive membranes and maintained for at least $8 \mathrm{~h}$. Manipulation of the LCV PI pattern is of great importance for the bacterium. The PI 5phosphatase Dd5P4 as well as PI3Ks and PI4KIII $\beta$ have been 
implicated in this process (Brombacher et al., 2009; Weber et al., 2009a,b).

Live-cell imaging was also employed to assess the LCV and microtubule dynamics in infected $D$. discoideum producing calnexin-GFP (Rothmeier et al., 2013) or GFP- $\alpha$-tubulin (Simon et al., 2014). The $D$. discoideum strains were infected with DsRed-producing $L$. pneumophila, wild-type, $\Delta l e g G 1$, or the complemented mutant. LegG1 is an Icm/Dot-translocated RCC1-like-repeat effector protein, which activates the small GTPase Ran and increases cellular RanGTP levels (Rothmeier et al., 2013). Ran is a member of the Ras superfamily of small GTPases and controls various cellular processes, including nucleo-cytoplasmic transport (Stewart, 2007), assembly of the mitotic spindle apparatus (Goodman and Zheng, 2006; Clarke and Zhang, 2008), and the dynamics of non-centrosomal microtubules (Yudin and Fainzilber, 2009). LCV motility as well as microtubule stability was significantly reduced in D. discoideum infected with $\Delta$ legG1 mutant bacteria.

\section{QUANTITATIVE ASSESSMENT OF LEGIONELLA INFECTION BY (IMAGING) FLOW CYTOMETRY}

Flow cytometry is a high-throughput technique that allows a simultaneous multi-parameter analysis of single cells. Large data sets, high resolution, accuracy, reproducibility, and sensitivity are major advantages of this approach to study uptake, cytotoxicity, and intracellular replication of L. pneumophila (Tiaden et al., 2013). The physiological status and integrity of a cell is reflected in the forward scatter/sideward scatter pattern (corresponding to cell size and "granularity," respectively), and membrane integrity can be tested by staining cellular DNA with the fluorescent dye propidium iodide, which is recorded as "cell-associated fluorescence."

Flow cytometry can be used to study in a quantitative manner Acanthamoeba phagocytosis. To this end, the rate and degree of uptake of fluorescent beads are assessed, i.e., the ratio of fluorescent vs. non-fluorescent amoebae (Avery et al., 1995), or, analogously, the infection by fluorescently labeled L. pneumophila is determined (Tiaden et al., 2013). Uptake of $L$. pneumophila is scored immediately after infection by determining an "uptake index," which is calculated from the signal-strength and percentage of amoebae infected with GFPproducing bacteria (Harf et al., 1997; Tiaden et al., 2013). Hence, cytotoxicity of $L$. pneumophila can be assessed as a change in size and shape of A. castellanii, measured by forward/sideward light scattering, as well as by loss of membrane integrity, as determined by the increase in permeability to propidium iodide (Tiaden et al., 2007, 2008) (Figure 2E).

Moreover, using flow cytometry and GFP-producing bacteria, not only the uptake efficiency can be determined, but also intracellular replication in A. castellanii or D. discoideum can be followed over several days. This approach has been utilized to examine the uptake and replication defects of $L$. pneumophila deletion mutants lacking components of the Legionella quorum sensing (Lqs) system: LqsR (Tiaden et al., 2007), LqsA or
LqsS (Tiaden et al., 2010), LqsT or LqsS-LqsT (Kessler et al., 2013), as well as the entire lqs gene cluster (Tiaden et al., 2008) (Figure 2F). Analogously, flow cytometry can be employed to assess uptake and replication phenotypes of $D$. discoideum deletion mutant strains lacking PI3Ks (Weber et al., 2006) or Dd5P4 (Weber et al., 2009a). Finally, in addition to its use as a research tool, flow cytometry has also been developed as a diagnostic method to rapidly screen for Legionella contamination of bathing facilities and other water sources (Taguri et al., 2011).

In a technique termed imaging flow cytometry (IFC), flow cytometry is combined with fluorescence microscopy, enabling fast quantification of microscopic images. Using this approach, the high-throughput capacity and information about cell size, volume, and shape provided by the flow cytometer is combined with high-resolution spatial localization of fluorescent proteins of interest provided by a microscopic image, resulting in nonbiased and quantitative, large datasets (Barteneva et al., 2012; Johansson et al., 2015). By means of specialized software, the acquired cellular images can be analyzed using bi-variant plots and histograms, in parallel with a sequential gating strategy.

Recently, we employed IFC and D. discoideum ectopically producing GFP- and mCherry-labeled fusion proteins to assess the accumulation of specific host proteins on LCVs in whole cells (Schmölders et al., 2017; Steiner et al., 2017a) or homogenates (Bärlocher et al., 2017a). Thus, active small GTPase Rap1 was found to localize to a higher extent to LCVs containing the parental L. pneumophila strain Lp02 as compared to LCVs harboring the "pentuple" mutant strain (Schmölders et al., 2017), which lacks five gene clusters encoding $31 \%$ of the effector proteins (O'Connor et al., 2011). The method was also utilized to quantify the recruitment of calnexin-GFP or GFP-Sey1 to PI(4)P-positive LCVs labeled with P4C-mCherry. Dually labeled $D$. discoideum amoebae producing P4C-mCherry and GFP-Sey1 or GFP-Sey1_K154A were infected, and colocalization of $\mathrm{PI}(4) P$ with $L$. pneumophila was analyzed for 10,000 events per time point p.i. IFC confirmed and quantified the fluorescence microscopy finding that $\mathrm{PI}(4) P$ accumulates at the LCV in a Sey1-independent manner. Finally, the Rab7 GAP TBC1D5 was scored on LCVs. However, in this case lysates of infected $D$. discoideum had to be prepared to reduce background and increase sensitivity (Bärlocher et al., 2017a). To this end, D. discoideum amoebae producing in tandem GFP-TBC1D5 and the endosomal marker AmtA-mCherry were infected with mPlum-producing L. pneumophila, and the localization of TBC1D5 was analyzed by IFC in cell homogenates. For each condition, over 3,000 cells were analyzed, and TBC1D5 was found to localize to wild-type-, but not $\Delta i c m T$-containing LCVs.

\section{CHEMOTACTIC MIGRATION OF LEGIONELLA-INFECTED D. DISCOIDEUM}

In a process termed chemotaxis, phagocytes migrate in a directed manner toward an attractant. Thus, D. discoideum, 
macrophages or neutrophils sense and respond to cAMP, the chemokines CCL5, and tumor necrosis factor (TNF)- $\alpha$, or the formyl-methionyl-leucyl-phenylalanine (fMLP) peptide, respectively. The migration of eukaryotic cells critically depends on microtubule polarization and dynamics (Etienne-Manneville, 2013), which in turn is controlled by the small GTPase Ran.

Given the impact of the Icm/Dot substrate LegG1 on Ran GTPase activation and microtubule dynamics (Rothmeier et al., 2013), we analyzed the role of the L. pneumophila T4SS and LegG1 on host cell motility (Simon et al., 2014). Using D. discoideum amoebae or mammalian immune cells in underagarose or Boyden chamber migration assays, L. pneumophila was found to inhibit cell migration in an Icm/Dot-dependent manner. D. discoideum infected with virulent $L$. pneumophila showed a substantially reduced migration when compared to amoebae infected with $\Delta i c m T$ mutant bacteria. The migration inhibition observed was not due to an uptake defect or cytotoxicity. Interestingly, $D$. discoideum infected with the $\Delta$ legG1 mutant strain was hyper-inhibited for directed migration in the under-agarose assay, which was reverted upon overproduction of LegG1 to an extent comparable to amoebae infected with wild-type bacteria. Single cell tracking indicated that the directionality as well as the velocity of $D$. discoideum infected with the parental strain or $\Delta \operatorname{leg} G 1$ is impaired. Thus, the Ran activator LegG1 promotes cell motility by stabilizing microtubules and thereby might antagonize the possibly deleterious impact of other L. pneumophila Icm/Dot substrates on the host cytoskeleton.

Recently, the effect of the small signaling molecule LAI1 (Legionella autoinducer-1, 3-hydroxypentadecane-4-one) on chemotactic migration of $D$. discoideum was analyzed (Simon et al., 2015). This study was based on the observations that L. pneumophila lacking the autoinducer synthase LqsA no longer impaired the migration of infected amoebae, and a $\Delta i c m T$ mutant strain overproducing LqsA inhibited cell migration, obviously in an Icm/Dot-independent manner. Synthetic LAI1 dose-dependently inhibited the migration of $D$. discoideum in the micromolar range. RNA interference with epithelial cells subjected to a scratch ("wound closure") assay revealed that LAI-1 signaling requires the scaffold protein IQGAP1, the small GTPase Cdc42 as well as the Cdc42-specific guanine nucleotide exchange factor ARHGEF9, but not other modulators of Cdc42, or RhoA, Racl or Ran GTPase. Thus, the L. pneumophila signaling molecule LAI-1 produced either in the LCV or added exogenously promotes inter-kingdom signaling.

In summary, L. pneumophila modulates the motility of eukaryotic cells not only by means of $\mathrm{Icm} /$ Dot substrates, but also through the Lqs system and the small signaling molecule LAI-1 (Personnic et al., 2017). At present, it is unclear what the benefit of host cell migration inhibition for L. pneumophila might be. The phenomenon might simply be an indirect effect of perturbation of trafficking pathways and cytoskeletal dynamics. More intriguingly, however, it might also reflect the fact that macropinocytosis competes with migration of $D$. discoideum, two processes balanced by $\mathrm{PI}(3,4,5) P_{3}$ (Veltman, 2015). Hence, by inhibiting cell migration, L. pneumophila might promote its macropinocytic uptake by the amoebae.

\section{CELL BIOLOGICAL, BIOCHEMICAL, AND PROTEOMICS ANALYSIS OF PURIFIED INTACT LCVS}

Intact LCVs from $D$. discoideum can be purified by a straight-forward two-step protocol involving immuno-affinity enrichment using an antibody against the $\mathrm{Icm} /$ Dot substrate SidC, which selectively decorates the pathogen vacuole, and a secondary antibody coupled to magnetic beads. This first step is followed by conventional Histodenz gradient density centrifugation, yielding intact LCVs in high purity and yield (Urwyler et al., 2010; Hoffmann et al., 2012, 2013) (Figure 2G). Using D. discoideum producing the LCV marker calnexinGFP, infected with red fluorescent L. pneumophila, the LCV purification procedure can be easily followed by fluorescence microscopy.

Proteomics analysis of these purified LCVs revealed the presence of 560-1150 host proteins (Urwyler et al., 2009; Hoffmann et al., 2014; Schmölders et al., 2017). Amoebae proteins identified include small Rab GTPases, Rap1, Ran, and its effector Ran binding protein 1 (RanBP1). Moreover, large GTPases, components of the endosomal and late secretory trafficking pathways, as well as protein or lipid kinases and phosphatases were identified, many of which had not been implicated in LCV formation before. The accumulation of several of these host proteins on the LCV was confirmed by fluorescence microscopy using $D$. discoideum strains producing the corresponding GFP-fusion proteins (Urwyler et al., 2009; Finsel et al., 2013a; Rothmeier et al., 2013; Hoffmann et al., 2014; Schmölders et al., 2017; Steiner et al., 2017a). While some of these host factors accumulate on LCVs in an Icm/Dot-dependent manner (e.g., Rab1,-7,-8,-14, Rap1, Ran, RanBP1, Sey1), others localize to the pathogen vacuole $\mathrm{Icm} /$ Dot-independently (e.g., retromer subunits).

Preparations of intact LCVs can also be utilized for biochemical experiments (Steiner et al., 2017a). To this end, D. discoideum producing calnexin-GFP, GFP-Sey1, or catalytically inactive GFP-Sey1_K154A were infected with mCherry-producing virulent $L$. pneumophila. LCVs were purified according to the two-step protocol described above, and subsequently, the effect of GTP, GDP or the non-hydrolysable GTP analog Gpp(NH)p was analyzed by fluorescence microscopy. Interestingly, the purified LCVs aggregated and expanded their size about 2-fold in a Sey1and GTP-dependent manner. This experiment revealed in a reductionist system the importance of Seyl activity for LCV aggregation and circumferential ER remodeling.

\section{SCREEN FOR ANTIVIRULENCE COMPOUNDS IN LEGIONELLA-INFECTED AMOEBAE}

Antibiotic resistance of human pathogens is an ever-rising and global problem. The approach to develop new bactericidal or bacteriostatic antibiotics is compromised by a high selection pressure on pathogens to become resistant against these 
compounds. A more efficient method is to target the virulence and the ability of bacteria to infect the host, rather than targeting directly the capacity to multiply. Such an "anti-virulence" strategy aims at specific mechanisms that promote bacterial pathogenicity, including metabolic pathways, binding to and uptake by host cells, signaling, and vesicle trafficking pathways, or the secretion and mode of action of toxins. There is the hope that anti-virulence compounds reduce the evolutionary pressure to develop drug resistance, and by selectively targeting virulent bacteria, spare the normal host microbiota.

Another major obstacle in developing novel antibiotic or antivirulence compounds is the limited compound accessibility of intracellular bacteria, reflected in compromised bioavailability of a compound. To efficiently address these problems, and given the similarities between amoebae and human immune phagocytes such as macrophages or neutrophils (Hilbi et al., 2007; Escoll et al., 2013; Hoffmann et al., 2013), we established A. castellanii as a robust and high-throughput compatible model system to screen for anti-virulence compounds. To this end, the amoebae were seeded into 96-well plates and infected with GFP-producing L. pneumophila. The progress of intracellular growth correlates with an increase in fluorescence (Harrison et al., 2013, 2015b). The medium chosen minimizes auto-fluorescence and growth of the amoebae, as well as extracellular growth of the bacteria. Under these conditions, bacterial replication typically comprised a lag and a replicative phase, after which all amoebae were dead and bacterial fluorescence remained constant. Upon addition of low molecular weight compounds to infected amoebae, we identified palmostatin $\mathrm{M}-\mathrm{a} \beta$-lactone inhibitor of eukaryotic Ras depalmitoylases and Ras signaling (Hedberg et al., 2011; Rusch et al., 2011) - as an inhibitor of intracellular growth of L. pneumophila as well as of Mycobacterium marinum and Mycobacterium tuberculosis (Harrison et al., 2013; Kicka et al., 2014).

Moreover, using this A. castellanii-L. pneumophila infection assay, we screened a highly diverse, pathway-based chemical library, referred to as the Sinergia library (Harrison et al., 2015a). Structure-activity relationship (SAR) studies using variants of a hit compound thus identified revealed essential structural features, namely a triple-ring scaffold with a central triazine moiety, substituted by two piperidine or pyrrolidine rings in positions 3 and 5, and by an amine group bearing a single aliphatic chain moiety in position 1 . The most effective compound, ZINC00615682, inhibited intracellular growth of L. pneumophila with an $\mathrm{IC}_{50}$ of $\sim 20 \mathrm{nM}$ in A. castellanii and somewhat less efficiently in $D$. discoideum or macrophages (Harrison et al., 2015a). These results validate the amoebae-based screen and demonstrate that a corresponding SAR analysis allows the identification of novel inhibitors of intracellularly growing L. pneumophila and other bacteria.

\section{REFERENCES}

Albers, U., Reus, K., Shuman, H. A., and Hilbi, H. (2005). The amoebae plate test implicates a paralogue of $\operatorname{lp} x B$ in the interaction of Legionella

\section{CONCLUSIONS AND OUTLOOK}

Intense research over the last years revealed that amoebae not only represent an important ecological niche of the environmental bacterium L. pneumophila, but are also versatile and powerful models for the molecular and cellular analysis of host-pathogen interactions. The natural host A. castellanii is permissive for $L$. pneumophila over a range of different temperatures, and this robust system is useful to assess bacterial mutant phenotypes, as well as to screen for anti-virulence compounds. The social amoeba $D$. discoideum is genetically tractable and allows analysis of host mutant strains. Dually labeled $D$. discoideum strains are ideally suited for (live-cell) fluorescence microscopy, imaging flow cytometry, cell migration assays, or LCV isolation. Purified intact LCVs can be further analyzed by proteomics and cell biological or biochemical tests. Obvious limitations of the amoebae models are the apparent lack of key components of cell-autonomous innate immunity pathways, such as caspase family proteases and the transcription factor NF-kB (Eichinger et al., 2005; Clarke et al., 2013). On the other hand, amoebae models are useful for the analysis of host-pathogen interactions beyond Legionella infection. Accordingly, Acanthamoeba spp. and D. discoideum can serve to assess various aspects of cellular virulence for a large number of pathogenic bacteria including Mycobacterium spp., Burkholderia spp., and Vibrio cholerae (Hilbi et al., 2007; Cosson and Soldati, 2008). Hence, amoebae and other protozoa will continue to provide valuable insights into important aspects of the ecology and virulence of amoeba-resistant environmental bacterial pathogens.

\section{AUTHOR CONTRIBUTIONS}

ALS, CFH, LE, MS, and HH wrote the manuscript.

\section{ACKNOWLEDGMENTS}

We would like to thank Stephen Weber for providing Figure 2D. Research in the laboratory of $\mathrm{HH}$ was supported by the Swiss National Science Foundation (SNF; 31003A_153200), the University of Zürich, the Novartis Foundation for MedicalBiological Research, the OPO foundation, and the German Ministry of Education and Research (BMBF) in the context of the EU Infect-ERA initiative (project EUGENPATH; 031A410A). LE acknowledges support of this work by the German Research Foundation (Deutsche Forschungsgemeinschaft, DFG: CRC670, TP01) and by Köln Fortune. Research in the laboratory of MS was supported by DFG STE 838/8-1. The funders had no role in study design, data collection and analysis, decision to publish, or preparation of the manuscript. 
A biosynthesis genes under different growth conditions. Microbiology 153, 3817-3829. doi: 10.1099/mic.0.2007/009829-0

Al-Quadan, T., and Kwaik, Y. A. (2011). Molecular characterization of exploitation of the polyubiquitination and farnesylation machineries of Dictyostelium discoideum by the AnkB F-Box effector of Legionella pneumophila. Front. Microbiol. 2:23. doi: 10.3389/fmicb.2011.00023

Amaro, F., Wang, W., Gilbert, J. A., Anderson, O. R., and Shuman, H. A. (2015). Diverse protist grazers select for virulence-related traits in Legionella. ISME J. 9, 1607-1618. doi: 10.1038/ismej.2014.248

Asrat, S., de Jesús, D. A., Hempstead, A. D., Ramabhadran, V., and Isberg, R. R. (2014). Bacterial pathogen manipulation of host membrane trafficking. Annu. Rev. Cell Dev. Biol. 30, 79-109. doi: 10.1146/annurev-cellbio-100913013439

Aurass, P., Pless, B., Rydzewski, K., Holland, G., Bannert, N., and Flieger, A. (2009). BdhA-patD operon as a virulence determinant, revealed by a novel large-scale approach for identification of Legionella pneumophila mutants defective for amoeba infection. Appl. Environ. Microbiol. 75, 4506-4515. doi: 10.1128/AEM.00187-09

Avery, S. V., Harwood, J. L., and Lloyd, D. (1995). Quantification and characterization of phagocytosis in the soil amoeba Acanthamoeba castellanii by flow cytometry. Appl. Environ. Microbiol. 61, 1124-1132.

Barker, J., Scaife, H., and Brown, M. R. (1995). Intraphagocytic growth induces an antibiotic-resistant phenotype of Legionella pneumophila. Antimicrob. Agents Chemother. 39, 2684-2688. doi: 10.1128/AAC.39.12.2684

Bärlocher, K., Hutter, C. A. J., Swart, A. L., Steiner, B., Welin, A., Hohl, M., et al. (2017a). Structural insights into Legionella RidL-Vps29 retromer subunit interaction reveal displacement of the regulator TBC1D5. Nat. Commun. 8:1543. doi: 10.1038/s41467-017-01512-5

Bärlocher, K., Welin, A., and Hilbi, H. (2017b). Formation of the Legionella replicative compartment at the crossroads of retrograde trafficking. Front. Cell. Infect. Microbiol. 7:482. doi: 10.3389/fcimb.2017.00482.

Barteneva, N. S., Fasler-Kan, E., and Vorobjev, I. A. (2012). Imaging flow cytometry: coping with heterogeneity in biological systems. J. Histochem. Cytochem. 60, 723-733. doi: 10.1369/0022155412453052

Benin, A. L., Benson, R. F., and Besser, R. E. (2002). Trends in Legionnaires' disease, 1980-1998: declining mortality and new patterns of diagnosis. Clin. Infect. Dis. 35, 1039-1046. doi: 10.1086/342903

Bergmann, S., and Steinert, M. (2015). From single cells to engineered and explanted tissues: new perspectives in bacterial infection biology. Int. Rev. Cell Mol. Biol. 319, 1-44. doi: 10.1016/bs.ircmb.2015.06.003

Berk, S. G., and Garduño, R. A. (2013). The Tetrahymena and Acanthamoeba model systems. Methods Mol. Biol. 954, 393-416. doi: 10.1007/978-1-62703-161-5_25

Bowers, B., and Korn, E. D. (1973). Cytochemical identification of phosphatase activity in the contractile vacuole of Acanthamoeba castellanii. J. Cell Biol. 59, 784-791. doi: 10.1083/jcb.59.3.784

Bozzaro, S., Bucci, C., and Steinert, M. (2008). Phagocytosis and host-pathogen interactions in Dictyostelium with a look at macrophages. Int. Rev. Cell Mol. Biol. 271, 253-300. doi: 10.1016/S1937-6448(08)01206-9

Bozzaro, S., and Eichinger, L. (2011). The professional phagocyte Dictyostelium discoideum as a model host for bacterial pathogens. Curr. Drug Targets 12, 942-954. doi: 10.2174/138945011795677782

Bozzaro, S., Peracino, B., and Eichinger, L. (2013). Dictyostelium host response to Legionella infection: strategies and assays. Methods Mol. Biol. 954, 417-438. doi: 10.1007/978-1-62703-161-5_26

Brassinga, A. K., Kinchen, J. M., Cupp, M. E., Day, S. R., Hoffman, P. S., and Sifri, C. D. (2010). Caenorhabditis is a metazoan host for Legionella. Cell. Microbiol. 12, 343-361. doi: 10.1111/j.1462-5822.2009.01398.x

Bretschneider, T., Othmer, H. G., and Weijer, C. J. (2016). Progress and perspectives in signal transduction, actin dynamics, and movement at the cell and tissue level: lessons from Dictyostelium. Interface Focus 6:20160047. doi: 10.1098/rsfs.2016.0047

Brieland, J., McClain, M., LeGendre, M., and Engleberg, C. (1997). Intrapulmonary Hartmannella vermiformis: a potential niche for Legionella pneumophila replication in a murine model of legionellosis. Infect. Immun. 65, 4892-4896.

Brombacher, E., Urwyler, S., Ragaz, C., Weber, S. S., Kami, K., Overduin, M., et al. (2009). Rab1 guanine nucleotide exchange factor SidM is a major phosphatidylinositol 4-phosphate-binding effector protein of Legionella pneumophila. J. Biol. Chem. 284, 4846-4856. doi: 10.1074/jbc.M807505200

Brown, T. J., and Cursons, R. T. (1977). Pathogenic free-living amebae (PFLA) from frozen swimming areas in Oslo, Norway. Scand. J. Infect. Dis. 9, 237-240. doi: 10.3109/inf.1977.9.issue-3.16

Brown, T. J., Cursons, R. T., and Keys, E. A. (1982). Amoebae from antarctic soil and water. Appl. Environ. Microbiol. 44, 491-493.

Brüggemann, H., Hagman, A., Jules, M., Sismeiro, O., Dillies, M. A., Gouyette, C., et al. (2006). Virulence strategies for infecting phagocytes deduced from the in vivo transcriptional program of Legionella pneumophila. Cell. Microbiol. 8, 1228-1240. doi: 10.1111/j.1462-5822.2006.00703.x

Cazalet, C., Gomez-Valero, L., Rusniok, C., Lomma, M., Dervins-Ravault, D., Newton, H. J., et al. (2010). Analysis of the Legionella longbeachae genome and transcriptome uncovers unique strategies to cause Legionnaires' disease. PLoS Genet. 6:e1000851. doi: 10.1371/journal.pgen.1000851

Cazalet, C., Rusniok, C., Brüggemann, H., Zidane, N., Magnier, A., Ma, L., et al. (2004). Evidence in the Legionella pneumophila genome for exploitation of host cell functions and high genome plasticity. Nat. Genet. 36, 1165-1173. doi: $10.1038 / \mathrm{ng} 1447$

Chang, C. W., Kao, C. H., and Liu, Y. F. (2009). Heterogeneity in chlorine susceptibility for Legionella pneumophila released from Acanthamoeba and Hartmannella. J. Appl. Microbiol. 106, 97-105. doi: 10.1111/j.1365-2672.2008.03980.x

Chávez-Munguía, B., Omaña-Molina, M., González-Robles, M., Gonzalez-Robles, A., Bonilla, P., and Martínez-Palomo, A. (2005). Ultrastructural study of encystation and excystation in Acanthamoeba castellanii. J. Eukaryot. Microbiol. 52, 153-158. doi: 10.1111/j.1550-7408.2005.04-3273.x

Chen, P., Ostrow, B. D., Tafuri, S. R., and Chisholm, R. L. (1994). Targeted disruption of the Dictyostelium RMLC gene produces cells defective in cytokinesis and development. J. Cell Biol. 127, 1933-1944. doi: $10.1083 /$ jcb.127.6.1933

Chien, M., Morozova, I., Shi, S., Sheng, H., Chen, J., Gomez, S. M., et al. (2004). The genomic sequence of the accidental pathogen Legionella pneumophila. Science 305, 1966-1968. doi: 10.1126/science.1099776

Choy, A., Dancourt, J., Mugo, B., O'connor, T. J., Isberg, R. R., Melia, T. J., et al. (2012). The Legionella effector RavZ inhibits host autophagy through irreversible Atg8 deconjugation. Science 338, 1072-1076. doi: 10.1126/science.1227026

Cirillo, J. D., Falkow, S., and Tompkins, L. S. (1994). Growth of Legionella pneumophila in Acanthamoeba castellanii enhances invasion. Infect. Immun. 62, 3254-3261.

Clarke, M., Lohan, A. J., Liu, B., Lagkouvardos, I., Roy, S., Zafar, N., et al. (2013). Genome of Acanthamoeba castellanii highlights extensive lateral gene transfer and early evolution of tyrosine kinase signaling. Genome Biol. 14:R11. doi: 10.1186/gb-2013-14-2-r11

Clarke, P. R., and Zhang, C. (2008). Spatial and temporal coordination of mitosis by Ran GTPase. Nat. Rev. Mol. Cell Biol. 9, 464-477. doi: 10.1038/nrm2410

Coil, D. A., Anné, J., and Lammertyn, E. (2008). A faster and more accurate assay for intracellular replication of Legionella pneumophila in amoebae hosts. J. Microbiol. Methods 72, 214-216. doi: 10.1016/j.mimet.2007.11.009

Correia, A. M., Ferreira, J. S., Borges, V., Nunes, A., Gomes, B., Capucho, R., et al. (2016). Probable person-to-person transmission of Legionnaires' disease. $N$. Engl. J. Med. 374, 497-498. doi: 10.1056/NEJMc1505356

Cosson, P., and Soldati, T. (2008). Eat, kill or die: when amoeba meets bacteria. Curr. Opin. Microbiol. 11, 271-276. doi: 10.1016/j.mib.2008.05.005

Di Paolo, G., and De Camilli, P. (2006). Phosphoinositides in cell regulation and membrane dynamics. Nature 443, 651-657. doi: 10.1038/nature05185

Dolinsky, S., Haneburger, I., Cichy, A., Hannemann, M., Itzen, A., and Hilbi, H. (2014). The Legionella longbeachae Icm/Dot substrate SidC selectively binds phosphatidylinositol 4-phosphate with nanomolar affinity and promotes pathogen vacuole-endoplasmic reticulum interactions. Infect. Immun. 82, 4021-4033. doi: 10.1128/IAI.01685-14

Eichinger, L., Pachebat, J. A., Glöckner, G., Rajandream, M. A., Sucgang, R., Berriman, M., et al. (2005). The genome of the social amoeba Dictyostelium discoideum. Nature 435, 43-57. doi: 10.1038/nature03481

Eichinger, L., and Rivero, F. (2006). Methods in Molecular Biology - Dictyostelium discoideum protocols. Totowa, NJ: Humana Press. 
Eisenreich, W., and Heuner, K. (2016). The life stage-specific pathometabolism of Legionella pneumophila. FEBS Lett. 590, 3868-3886. doi: $10.1002 / 1873-3468.12326$

Escoll, P., Rolando, M., Gomez-Valero, L., and Buchrieser, C. (2013). From amoeba to macrophages: exploring the molecular mechanisms of Legionella pneumophila infection in both hosts. Curr. Top. Microbiol. Immunol. 376, 1-34. doi: 10.1007/82_2013_351

Etienne-Manneville, S. (2013). Microtubules in cell migration. Annu. Rev. Cell Dev. Biol. 29, 471-499. doi: 10.1146/annurev-cellbio-101011-155711

Faix, J., Kreppel, L., Shaulsky, G., Schleicher, M., and Kimmel, A. R. (2004). A rapid and efficient method to generate multiple gene disruptions in Dictyostelium discoideum using a single selectable marker and the Cre-loxP system. Nucleic Acids Res. 32:e143. doi: 10.1093/nar/gnh136

Fajardo, M., Schleicher, M., Noegel, A., Bozzaro, S., Killinger, S., Heuner, K., et al. (2004). Calnexin, calreticulin and cytoskeleton-associated proteins modulate uptake and growth of Legionella pneumophila in Dictyostelium discoideum. Microbiology 150, 2825-2835. doi: 10.1099/mic.0.27111-0

Farbrother, P., Wagner, C., Na, J., Tunggal, B., Morio, T., Urushihara, H., et al. (2006). Dictyostelium transcriptional host cell response upon infection with Legionella. Cell. Microbiol. 8, 438-456. doi: 10.1111/j.1462-5822.2005. 00633.x

Fey, P., Dodson, R. J., Basu, S., and Chisholm, R. L. (2013). One stop shop for everything Dictyostelium: dictyBase and the Dicty Stock Center in 2012. Methods Mol. Biol. 983, 59-92. doi: 10.1007/978-1-62703-302-2_4

Fields, B. S. (1996). The molecular ecology of legionellae. Trends Microbiol. 4, 286-290. doi: 10.1016/0966-842X(96)10041-X

Finsel, I., and Hilbi, H. (2015). Formation of a pathogen vacuole according to Legionella pneumophila: how to kill one bird with many stones. Cell. Microbiol. 17, 935-950. doi: $10.1111 / \mathrm{cmi} .12450$

Finsel, I., Hoffmann, C., and Hilbi, H. (2013a). Immunomagnetic purification of fluorescent Legionella-containing vacuoles. Methods Mol. Biol. 983, 431-443. doi: 10.1007/978-1-62703-302-2_24

Finsel, I., Ragaz, C., Hoffmann, C., Harrison, C. F., Weber, S., Van Rahden, V. A., et al. (2013b). The Legionella effector RidL inhibits retrograde trafficking to promote intracellular replication. Cell Host Microbe 14, 38-50. doi: 10.1016/j.chom.2013.06.001

Gaudet, P., Fey, P., Basu, S., Bushmanova, Y. A., Dodson, R., Sheppard, K. A., et al. (2011). DictyBase update 2011: web 2.0 functionality and the initial steps towards a genome portal for the Amoebozoa. Nucleic Acids Res. 39, D620-D624. doi: 10.1093/nar/gkq1103

Goodman, B., and Zheng, Y. (2006). Mitotic spindle morphogenesis: Ran on the microtubule cytoskeleton and beyond. Biochem. Soc. Trans. 34, 716-721. doi: $10.1042 /$ BST0340716

Greub, G., and Raoult, D. (2004). Microorganisms resistant to free-living amoebae. Clin. Microbiol. Rev. 17, 413-433. doi: 10.1128/CMR.17.2.413-433.2004

Hägele, S., Köhler, R., Merkert, H., Schleicher, M., Hacker, J., and Steinert, M. (2000). Dictyostelium discoideum: a new host model system for intracellular pathogens of the genus Legionella. Cell. Microbiol. 2, 165-171. doi: 10.1046/j.1462-5822.2000.00044.x

Hall-Stoodley, L., Costerton, J. W., and Stoodley, P. (2004). Bacterial biofilms: from the natural environment to infectious diseases. Nat. Rev. Microbiol. 2, 95-108. doi: $10.1038 /$ nrmicro821

Haneburger, I., and Hilbi, H. (2013). Phosphoinositide lipids and the Legionella pathogen vacuole. Curr. Top. Microbiol. Immunol. 376, 155-173. doi: 10.1007/82_2013_341

Harf, C., Goffinet, S., Meunier, O., Monteil, H., and Colin, D. A. (1997). Flow cytometric determination of endocytosis of viable labelled Legionella pneumophila by Acanthamoeba palestinensis. Cytometry 27, 269-274. doi: 10. 1002/(SICI) 1097-0320(19970301)27:3<269::AID-CYTO9>3.0.CO;2-9

Harrison, C. F., Chiriano, G., Finsel, I., Manske, C., Hoffmann, C., Steiner, B., et al. (2015a). Amoebae-based screening reveals a novel family of compounds restricting intracellular Legionella pneumophila. ACS Inf. Dis. 1, 327-338. doi: 10.1021/acsinfecdis.5b00002

Harrison, C. F., Kicka, S., Kranjc, A., Finsel, I., Chiriano, G., Ouertatani-Sakouhi, H., et al. (2015b). Adrenergic antagonists restrict replication of Legionella. Microbiology 161, 1392-1406. doi: 10.1099/mic.0.000094
Harrison, C. F., Kicka, S., Trofimov, V., Berschl, K., OuertataniSakouhi, H., Ackermann, N., et al. (2013). Exploring anti-bacterial compounds against intracellular Legionella. PLoS ONE 8:e74813. doi: 10.1371/journal.pone. 0074813

Hausmann, C. D., and Ibba, M. (2008). Aminoacyl-tRNA synthetase complexes: molecular multitasking revealed. FEMS Microbiol. Rev. 32, 705-721. doi: 10.1111/j.1574-6976.2008.00119.x

Hedberg, C., Dekker, F. J., Rusch, M., Renner, S., Wetzel, S., Vartak, N., et al. (2011). Development of highly potent inhibitors of the Ras-targeting human acyl protein thioesterases based on substrate similarity design. Angew. Chem. Int. Ed Engl. 50, 9832-9837. doi: 10.1002/anie.201102965

Hilbi, H., and Haas, A. (2012). Secretive bacterial pathogens and the secretory pathway. Traffic 13, 1187-1197. doi: 10.1111/j.1600-0854.2012.01344.x

Hilbi, H., Hoffmann, C., and Harrison, C. F. (2011). Legionella spp. outdoors: colonization, communication and persistence. Environ. Microbiol. Rep. 3, 286-296. doi: 10.1111/j.1758-2229.2011.00247.x

Hilbi, H., Weber, S. S., Ragaz, C., Nyfeler, Y., and Urwyler, S. (2007). Environmental predators as models for bacterial pathogenesis. Environ. Microbiol. 9, 563-575. doi: 10.1111/j.1462-2920.2007.01238.x

Hochstrasser, R., and Hilbi, H. (2017). Intra-species and inter-kingdom signaling of Legionella pneumophila. Front. Microbiol. 8:79. doi: 10.3389/fmicb.2017. 00079

Hoffmann, C., Finsel, I., and Hilbi, H. (2012). Purification of pathogen vacuoles from Legionella-infected phagocytes. J. Vis. Exp. e4118. doi: 10.3791/4118

Hoffmann, C., Finsel, I., and Hilbi, H. (2013). Pathogen vacuole purification from Legionella-infected amoeba and macrophages. Methods Mol. Biol. 954, 309-321. doi: 10.1007/978-1-62703-161-5_18

Hoffmann, C., Finsel, I., Otto, A., Pfaffinger, G., Rothmeier, E., Hecker, M., et al. (2014). Functional analysis of novel Rab GTPases identified in the proteome of purified Legionella-containing vacuoles from macrophages. Cell. Microbiol. 16, 1034-1052. doi: $10.1111 / \mathrm{cmi} .12256$

Hsu, T. K., Wu, S. F., Hsu, B. M., Kao, P. M., Tao, C. W., Shen, S. M., et al. (2015). Surveillance of parasitic Legionella in surface waters by using immunomagnetic separation and amoebae enrichment. Pathog. Glob. Health 109, 328-335. doi: $10.1179 / 2047773215 Y .0000000034$

Hubber, A., and Roy, C. R. (2010). Modulation of host cell function by Legionella pneumophila type IV effectors. Annu. Rev. Cell Dev. Biol. 26, 261-283. doi: 10.1146/annurev-cellbio-100109-104034

Isberg, R. R., O'connor, T. J., and Heidtman, M. (2009). The Legionella pneumophila replication vacuole: making a cosy niche inside host cells. Nat. Rev. Microbiol. 7, 13-24. doi: 10.1038/nrmicro1967

Jäger, J., Marwitz, S., Tiefenau, J., Rasch, J., Shevchuk, O., Kugler, C., et al. (2014). Human lung tissue explants reveal novel interactions during Legionella pneumophila infections. Infect. Immun. 82, 275-285. doi: 10.1128/IAI.00703-13

Johansson, J., Karlsson, A., Bylund, J., and Welin, A. (2015). Phagocyte interactions with Mycobacterium tuberculosis-Simultaneous analysis of phagocytosis, phagosome maturation and intracellular replication by imaging flow cytometry. J. Immunol. Methods 427, 73-84. doi: 10.1016/j.jim.2015.10.003

John, D. T., and Howard, M. J. (1995). Seasonal distribution of pathogenic free-living amebae in Oklahoma waters. Parasitol. Res. 81, 193-201.

Kessin, R. H., Gundersen, G. G., Zaydfudim, V., and Grimson, M. (1996). How cellular slime molds evade nematodes. Proc. Natl. Acad. Sci. U.S.A. 93, 4857-4861. doi: 10.1073/pnas.93.10.4857

Kessler, A., Schell, U., Sahr, T., Tiaden, A., Harrison, C., Buchrieser, C., et al. (2013). The Legionella pneumophila orphan sensor kinase LqsT regulates competence and pathogen-host interactions as a component of the LAI-1 circuit. Environ. Microbiol. 15, 646-662. doi: 10.1111/j.1462-2920.2012.02889.x

Kicka, S., Trofimov, V., Harrison, C., Ouertatani-Sakouhi, H., McKinney, J., Scapozza, L., et al. (2014). Establishment and validation of whole-cell based fluorescence assays to identify anti-mycobacterial compounds using the Acanthamoeba castellanii-Mycobacterium marinum host-pathogen system. PLoS ONE 9:e87834. doi: 10.1371/journal.pone.0087834

Komura, T., Yasui, C., Miyamoto, H., and Nishikawa, Y. (2010). Caenorhabditis elegans as an alternative model host for Legionella pneumophila, and protective effects of Bifidobacterium infantis. Appl. Environ. Microbiol. 76, 4105-4108. doi: 10.1128/AEM.03021-09 
Kuhlmann, M., Popova, B., and Nellen, W. (2006). RNA interference and antisense-mediated gene silencing in Dictyostelium. Methods Mol. Biol. 346, 211-226. doi: 10.1385/1-59745-144-4:211

Kuspa, A., and Loomis, W. F. (1992). Tagging developmental genes in Dictyostelium by restriction enzyme-mediated integration of plasmid DNA. Proc. Natl. Acad. Sci. U.S.A. 89, 8803-8807. doi: 10.1073/pnas.89.18.8803

Levi, S., Polyakov, M., and Egelhoff, T. T. (2000). Green fluorescent protein and epitope tag fusion vectors for Dictyostelium discoideum. Plasmid 44, 231-238. doi: $10.1006 /$ plas.2000.1487

Li, Z., Dugan, A. S., Bloomfield, G., Skelton, J., Ivens, A., Losick, V., et al. (2009). The amoebal MAP kinase response to Legionella pneumophila is regulated by DupA. Cell Host Microbe 6, 253-267. doi: 10.1016/j.chom.2009.08.005

Lorenzo-Morales, J., Ortega-Rivas, A., Foronda, P., Abreu-Acosta, N., Ballart, D., Martinez, E., et al. (2005). RNA interference (RNAi) for the silencing of extracellular serine proteases genes in Acanthamoeba: molecular analysis and effect on pathogenicity. Mol. Biochem. Parasitol. 144, 10-15. doi: 10.1016/j.molbiopara.2005.07.001

Lurie-Weinberger, M. N., Gomez-Valero, L., Merault, N., Glöckner, G., Buchrieser, C., and Gophna, U. (2010). The origins of eukaryotic-like proteins in Legionella pneumophila. Int. J. Med. Microbiol. 300, 470-481. doi: 10.1016/j.ijmm.2010.04.016

Manstein, D. J., Schuster, H. P., Morandini, P., and Hunt, D. M. (1995). Cloning vectors for the production of proteins in Dictyostelium discoideum. Gene 162, 129-134. doi: 10.1016/0378-1119(95)00351-6

Miyamoto, H., Yoshida, S., Taniguchi, H., and Shuman, H. A. (2003). Virulence conversion of Legionella pneumophila by conjugal transfer of chromosomal DNA. J. Bacteriol. 185, 6712-6718. doi: 10.1128/JB.185.22.6712-6718.2003

Moffat, J. F., and Tompkins, L. S. (1992). A quantitative model of intracellular growth of Legionella pneumophila in Acanthamoeba castellanii. Infect. Immun. 60, 296-301.

Molmeret, M., Horn, M., Wagner, M., Santic, M., and Abu Kwaik, Y. (2005). Amoebae as training grounds for intracellular bacterial pathogens. Appl. Environ. Microbiol. 71, 20-28. doi: 10.1128/AEM.71.1.20-28.2005

Müller-Taubenberger, A., Kortholt, A., and Eichinger, L. (2013). Simple systemsubstantial share: the use of Dictyostelium in cell biology and molecular medicine. Eur. J. Cell Biol. 92, 45-53. doi: 10.1016/j.ejcb.2012.10.003

. Newton, H. J., Ang, D. K., Van Driel, I. R., and Hartland, E. L. (2010). Molecular pathogenesis of infections caused by Legionella pneumophila. Clin. Microbiol. Rev. 23, 274-298. doi: 10.1128/CMR.00052-09

Noda, T. (2017). Autophagy in the context of the cellular membrane-trafficking system: the enigma of Atg9 vesicles. Biochem. Soc. Trans. 45, 1323-1331. doi: 10.1042/BST20170128

O’Connor, T. J., Adepoju, Y., Boyd, D., and Isberg, R. R. (2011). Minimization of the Legionella pneumophila genome reveals chromosomal regions involved in host range expansion. Proc. Natl. Acad. Sci. U.S.A. 108, 14733-14740. doi: $10.1073 /$ pnas.1111678108

Otto, G. P., Wu, M. Y., Clarke, M., Lu, H., Anderson, O. R., Hilbi, H., et al. (2004). Macroautophagy is dispensable for intracellular replication of Legionella pneumophila in Dictyostelium discoideum. Mol. Microbiol. 51, 63-72. doi: 10.1046/j.1365-2958.2003.03826.x

Peng, Z., Omaruddin, R., and Bateman, E. (2005). Stable transfection of Acanthamoeba castellanii. Biochim. Biophys. Acta 1743, 93-100. doi: 10.1016/j.bbamcr.2004.08.014

Peracino, B., Balest, A., and Bozzaro, S. (2010). Phosphoinositides differentially regulate bacterial uptake and Nrampl-induced resistance to Legionella infection in Dictyostelium. J. Cell Sci. 123, 4039-4051. doi: 10.1242/jcs.072124

Personnic, N., Bärlocher, K., Finsel, I., and Hilbi, H. (2016). Subversion of retrograde trafficking by translocated pathogen effectors. Trends Microbiol. 24, 450-462. doi: 10.1016/j.tim.2016.02.003

Personnic, N., Striednig, B., and Hilbi, H. (2017). Legionella quorum sensing and its role in pathogen-host interactions. Curr. Opin. Microbiol. 41, 29-35. doi: 10.1016/j.mib.2017.11.010

Pollard, T. D., Shelton, E., Weihing, R. R., and Korn, E. D. (1970). Ultrastructural characterization of F-actin isolated from Acanthamoeba castellanii and identification of cytoplasmic filaments as F-actin by reaction with rabbit heavy meromyosin. J. Mol. Biol. 50, 91-97. doi: 10.1016/0022-2836(70)90106-3
Preston, T. M., and King, C. A. (1984). Amoeboid locomotion of Acanthamoeba castellanii with special reference to cell-substratum interactions. J. Gen. Microbiol. 130, 2317-2323. doi: 10.1099/00221287-130-9-2317

Qiu, J., and Luo, Z. Q. (2017). Legionella and Coxiella effectors: strength in diversity and activity. Nat. Rev. Microbiol. 15, 591-605. doi: 10.1038/nrmicro.2017.67

Ragaz, C., Pietsch, H., Urwyler, S., Tiaden, A., Weber, S. S., and Hilbi, H. (2008). The Legionella pneumophila phosphatidylinositol-4 phosphatebinding type IV substrate SidC recruits endoplasmic reticulum vesicles to a replication-permissive vacuole. Cell. Microbiol. 10, 2416-2433. doi: 10.1111/j.1462-5822.2008.01219.x

Rasch, J., Krüger, S., Fontvieille, D., Ünal, C. M., Michel, R., Labrosse, A., et al. (2016). Legionella-protozoa-nematode interactions in aquatic biofilms and influence of Mip on Caenorhabditis elegans colonization. Int. J. Med. Microbiol. 306, 443-451. doi: 10.1016/j.ijmm.2016.05.012

Riyahi, T. Y., Frese, F., Steinert, M., Omosigho, N. N., Glöckner, G., Eichinger, L., et al. (2011). RpkA, a highly conserved GPCR with a lipid kinase domain, has a role in phagocytosis and anti-bacterial defense. PLoS ONE 6:e27311. doi: 10.1371/journal.pone.0027311

Rodriguez-Zaragoza, S., Rivera, F., Bonilla, P., Ramirez, E., Gallegos, E., Calderon, A., et al. (1993). Amoebological study of the atmosphere of San Luis Potosi, SLP, Mexico. J. Exp. Anal. Environ. Epidemiol. 3(Suppl. 1.), 229-241.

Rolando, M., Escoll, P., Nora, T., Botti, J., Boitez, V., Bedia, C., et al. (2016). Legionella pneumophila S1P-lyase targets host sphingolipid metabolism and restrains autophagy. Proc. Natl. Acad. Sci. U.S.A. 113, 1901-1906. doi: $10.1073 /$ pnas.1522067113

Romby, P., Vandenesch, F., and Wagner, E. G. (2006). The role of RNAs in the regulation of virulence-gene expression. Curr. Opin. Microbiol. 9, 229-236. doi: 10.1016/j.mib.2006.02.005

Rothmeier, E., Pfaffinger, G., Hoffmann, C., Harrison, C. F., Grabmayr, H., Repnik, U., et al. (2013). Activation of Ran GTPase by a Legionella effector promotes microtubule polymerization, pathogen vacuole motility and infection. PLoS Pathog. 9:e1003598. doi: 10.1371/journal.ppat.1003598

Rowbotham, T. J. (1986). Current views on the relationships between amoebae, legionellae and man. Isr. J. Med. Sci. 22, 678-689.

Rusch, M., Zimmermann, T. J., Bürger, M., Dekker, F. J., Görmer, K., Triola, G., et al. (2011). Identification of acyl protein thioesterases 1 and 2 as the cellular targets of the Ras-signaling modulators palmostatin B and M. Angew. Chem. Int. Ed Engl. 50, 9838-9842. doi: 10.1002/anie.201102967

Sakamoto, R., Ohno, A., Nakahara, T., Satomura, K., Iwanaga, S., Kouyama, Y., et al. (2009). Legionella pneumophila in rainwater on roads. Emerging Infect. Dis. 15, 1295-1297. doi: 10.3201/eid1508.090317

Sanden, G. N., Morrill, W. E., Fields, B. S., Breiman, R. F., and Barbaree, J. M. (1992). Incubation of water samples containing amoebae improves detection of legionellae by the culture method. Appl. Environ. Microbiol. 58, 2001-2004.

Sawyer, T. K. (1989). Free-living pathogenic and nonpathogenic amoebae in Maryland soils. Appl. Environ. Microbiol. 55, 1074-1077.

Schilde, C., Lawal, H. M., Noegel, A. A., Eichinger, L., Schaap, P., and Glöckner, G. (2016). A set of genes conserved in sequence and expression traces back the establishment of multicellularity in social amoebae. BMC Genomics 17:871. doi: 10.1186/s12864-016-3223-z

Schlimme, W., M., Marchiani, M., Hanselmann, K., and Jenni, B. (1997). Gene transfer between bacteria within digestive vacuoles of protozoa. FEMS Microbiol. Ecol. 23, 239-247. doi: 10.1111/j.1574-6941.1997.tb00406.x

Schmölders, J., Manske, C., Otto, A., Hoffmann, C., Steiner, B., Welin, A., et al. (2017). Comparative proteomics of purified pathogen vacuoles correlates intracellular replication of Legionella pneumophila with the small GTPase Ras-related protein 1 (Rap1). Mol. Cell. Proteomics 16, 622-641. doi: 10.1074/mcp.M116.063453

Schuster, F. L., and Levandowsky, M. (1996). Chemosensory responses of Acanthamoeba castellanii: visual analysis of random movement and responses to chemical signals. J. Eukaryot. Microbiol. 43, 150-158. doi: 10.1111/j.1550-7408.1996.tb04496.x

Segal, G., Purcell, M., and Shuman, H. A. (1998). Host cell killing and bacterial conjugation require overlapping sets of genes within a $22-\mathrm{kb}$ region of the Legionella pneumophila genome. Proc. Natl. Acad. Sci. U.S.A. 95, 1669-1674. doi: $10.1073 /$ pnas.95.4.1669 
Segal, G., and Shuman, H. A. (1999). Legionella pneumophila utilizes the same genes to multiply within Acanthamoeba castellanii and human macrophages. Infect. Immun. 67, 2117-2124.

Sherwood, R. K., and Roy, C. R. (2013). A Rab-centric perspective of bacterial pathogen-occupied vacuoles. Cell Host Microbe 14, 256-268. doi: 10.1016/j.chom.2013.08.010

Sherwood, R. K., and Roy, C. R. (2016). Autophagy evasion and endoplasmic reticulum subversion: the yin and yang of Legionella intracellular infection. Annu. Rev. Microbiol. 70, 413-433. doi: 10.1146/annurev-micro-102215-095557

Shevchuk, O., Pägelow, D., Rasch, J., Döhrmann, S., Günther, G., Hoppe, J., et al. (2014). Polyketide synthase (PKS) reduces fusion of Legionella pneumophila-containing vacuoles with lysosomes and contributes to bacterial competitiveness during infection. Int. J. Med. Microbiol. 304, 1169-1181. doi: 10.1016/j.ijmm.2014.08.010

Shina, M. C., Müller-Taubenberger, A., Unal, C., Schleicher, M., Steinert, M., Eichinger, L., et al. (2011). Redundant and unique roles of coronin proteins in Dictyostelium. Cell. Mol. Life Sci. 68, 303-313. doi: 10.1007/s00018-010-0455-y

Shina, M. C., and Noegel, A. A. (2008). Invertebrate coronins. Subcell. Biochem. 48, 88-97. doi: 10.1007/978-0-387-09595-0_8

Shina, M. C., Unal, C., Eichinger, L., Müller-Taubenberger, A., Schleicher, M., Steinert, M., et al. (2010). A coronin7 homolog with functions in actin-driven processes. J. Biol. Chem. 285, 9249-9261. doi: 10.1074/jbc.M109.083725

Simon, S., Schell, U., Heuer, N., Hager, D., Albers, M. F., Matthias, J., et al. (2015). Inter-kingdom signaling by the Legionella quorum sensing molecule LAI-1 modulates cell migration through an IQGAP1-Cdc42-ARHGEF9-dependent pathway. PLoS Pathog. 11:e1005307. doi: 10.1371/journal.ppat.1005307

Simon, S., Wagner, M. A., Rothmeier, E., Müller-Taubenberger, A., and Hilbi, H. (2014). Icm/Dot-dependent inhibition of phagocyte migration by Legionella is antagonized by a translocated Ran GTPase activator. Cell. Microbiol. 16, 977-992. doi: 10.1111/cmi.12258.

Solomon, J. M., Rupper, A., Cardelli, J. A., and Isberg, R. R. (2000). Intracellular growth of Legionella pneumophila in Dictyostelium discoideum, a system for genetic analysis of host-pathogen interactions. Infect. Immun. 68, 2939-2947. doi: 10.1128/IAI.68.5.2939-2947.2000

Steiner, B., Swart, A. L., Welin, A., Weber, S., Personnic, N., Kaech, A., et al. (2017a). ER remodeling by the large GTPase atlastin promotes vacuolar growth of Legionella pneumophila. EMBO Rep. 18, 1817-1836. doi: 10.15252/embr.201743903

Steiner, B., Weber, S., and Hilbi, H. (2017b). Formation of the Legionellacontaining vacuole: phosphoinositide conversion, GTPase modulation and ER dynamics. Int. J. Med. Microbiol. 308, 49-57. doi: 10.1016/j.ijmm.2017.08.004

Steinert, M. (2011). Pathogen-host interactions in Dictyostelium, Legionella, Mycobacterium and other pathogens. Semin. Cell Dev. Biol. 22, 70-76. doi: $10.1016 /$ j.semcdb.2010.11.003

Steinert, M., and Heuner, K. (2005). Dictyostelium as host model for pathogenesis. Cell. Microbiol. 7, 307-314. doi: 10.1111/j.1462-5822.2005.00493.x

Stewart, M. (2007). Molecular mechanism of the nuclear protein import cycle. Nat. Rev. Mol. Cell Biol. 8, 195-208. doi: 10.1038/nrm2114

Swaminathan, K., Stumpf, M., Müller, R., Horn, A. C., Schmidbauer, J., Eichinger, L., et al. (2015). Coronin7 regulates WASP and SCAR through CRIB mediated interaction with Rac proteins. Sci. Rep. 5:14437. doi: 10.1038/srep14437

Taguri, T., Oda, Y., Sugiyama, K., Nishikawa, T., Endo, T., Izumiyama, S., et al. (2011). A rapid detection method using flow cytometry to monitor the risk of Legionella in bath water. J. Microbiol. Methods 86, 25-32. doi: 10.1016/j.mimet.2011.03.012

Taylor, M., Ross, K., and Bentham, R. (2009). Legionella, protozoa, and biofilms: interactions within complex microbial systems. Microb. Ecol. 58, 538-547. doi: 10.1007/s00248-009-9514-Z

Tiaden, A. N., Kessler, A., and Hilbi, H. (2013). Analysis of Legionella infection by flow cytometry. Methods Mol. Biol. 954, 233-249. doi: 10.1007/978-1-62703-161-5_14

Tiaden, A., Spirig, T., Carranza, P., Brüggemann, H., Riedel, K., Eberl, L., et al. (2008). Synergistic contribution of the Legionella pneumophila lqs genes to pathogen-host interactions. J. Bacteriol. 190, 7532-7547. doi: 10.1128/JB.01002-08
Tiaden, A., Spirig, T., Sahr, T., Wälti, M. A., Boucke, K., Buchrieser, C., et al. (2010). The autoinducer synthase LqsA and putative sensor kinase LqsS regulate phagocyte interactions, extracellular filaments and a genomic island of Legionella pneumophila. Environ. Microbiol. 12, 1243-1259. doi: 10.1111/j.1462-2920.2010.02167.x

Tiaden, A., Spirig, T., Weber, S. S., Brüggemann, H., Bosshard, R., Buchrieser, C., et al. (2007). The Legionella pneumophila response regulator LqsR promotes host cell interactions as an element of the virulence regulatory network controlled by RpoS and LetA. Cell. Microbiol. 9, 2903-2920. doi: 10.1111/j.1462-5822.2007.01005.x

Tung, S. M., Unal, C., Ley, A., Peña, C., Tunggal, B., Noegel, A. A., et al. (2010). Loss of Dictyostelium Atg9 results in a pleiotropic phenotype affecting growth, development, phagocytosis and clearance and replication of Legionella pneumophila. Cell. Microbiol. 12, 765-780. doi: 10.1111/j.1462-5822.2010.01432.x

Ulsamer, A. G., Wright, P. L., Wetzel, M. G., and Korn, E. D. (1971). Plasma and phagosome membranes of Acanthamoeba castellanii. J. Cell Biol. 51, 193-215. doi: $10.1083 /$ jcb.51.1.193

Urwyler, S., Finsel, I., Ragaz, C., and Hilbi, H. (2010). Isolation of Legionellacontaining vacuoles by immuno-magnetic separation. Curr. Protoc. Cell Biol. Chapter 3, Unit 3 34. doi: 10.1002/0471143030.cb0334s46

Urwyler, S., Nyfeler, Y., Ragaz, C., Lee, H., Mueller, L. N., Aebersold, R., et al. (2009). Proteome analysis of Legionella vacuoles purified by magnetic immunoseparation reveals secretory and endosomal GTPases. Traffic 10, 76-87. doi: 10.1111/j.1600-0854.2008.00851.x

Veltman, D. M. (2015). Drink or drive: competition between macropinocytosis and cell migration. Biochem. Soc. Trans. 43, 129-132. doi: 10.1042/BST20140251

Veltman, D. M., and van Haastert, P. J. (2013). Extrachromosomal inducible expression. Methods Mol. Biol. 983, 269-281. doi: 10.1007/978-1-62703-302-2_14

Vogel, J. P., Andrews, H. L., Wong, S. K., and Isberg, R. R. (1998). Conjugative transfer by the virulence system of Legionella pneumophila. Science 279, 873-876. doi: 10.1126/science.279.5352.873

Weber, S., and Hilbi, H. (2014). Live-cell imaging of phosphoinositide dynamics during Legionella infection. Methods Mol. Biol. 1197, 153-167. doi: 10.1007/978-1-4939-1261-2_9

Weber, S. S., Ragaz, C., and Hilbi, H. (2009a). The inositol polyphosphate 5phosphatase OCRL1 restricts intracellular growth of Legionella, localizes to the replicative vacuole and binds to the bacterial effector LpnE. Cell. Microbiol. 11, 442-460. doi: 10.1111/j.1462-5822.2008.01266.x

Weber, S. S., Ragaz, C., and Hilbi, H. (2009b). Pathogen trafficking pathways and host phosphoinositide metabolism. Mol. Microbiol. 71, 1341-1352. doi: 10.1111/j.1365-2958.2009.06608.x

Weber, S. S., Ragaz, C., Reus, K., Nyfeler, Y., and Hilbi, H. (2006). Legionella pneumophila exploits $\mathrm{PI}(4) \mathrm{P}$ to anchor secreted effector proteins to the replicative vacuole. PLoS Pathog. 2:e46. doi: 10.1371/journal.ppat.0020046

Weber, S., Stirnimann, C. U., Wieser, M., Frey, D., Meier, R., Engelhardt, S., et al. (2014a). A type IV-translocated Legionella cysteine phytase counteracts intracellular growth restriction by phytate. J. Biol. Chem. 289, 34175-34188. doi: 10.1074/jbc.M114.592568

Weber, S., Wagner, M., and Hilbi, H. (2014b). Live-cell imaging of phosphoinositide dynamics and membrane architecture during Legionella infection. MBio 5, e00839-e00813. doi: 10.1128/mBio.00839-13

Weissenmayer, B. A., Prendergast, J. G., Lohan, A. J., and Loftus, B. J. (2011). Sequencing illustrates the transcriptional response of Legionella pneumophila during infection and identifies seventy novel small non-coding RNAs. PLoS ONE 6:e17570. doi: 10.1371/journal.pone.0017570

Wiegand, S., Kruse, J., Gronemann, S., and Hammann, C. (2011). Efficient generation of gene knockout plasmids for Dictyostelium discoideum using one-step cloning. Genomics 97, 321-325. doi: 10.1016/j.ygeno.2011.02.001

Xiong, Q., Ünal, C., Matthias, J., Steinert, M., and Eichinger, L. (2015). The phenotypes of Atg9, Atg16 and Atg9/16 knock-out mutants imply autophagy-dependent and -independent functions. Open Biol. 5:150008. doi: 10.1098/rsob.150008

Yamaguchi, N., Tokunaga, Y., Goto, S., Fujii, Y., Banno, F., and Edagawa, A. (2017). Rapid on-site monitoring of Legionella pneumophila in cooling 
tower water using a portable microfluidic system. Sci. Rep. 7:3092. doi: 10.1038/s41598-017-03293-9

Yannay-Cohen, N., Carmi-Levy, I., Kay, G., Yang, C. M., Han, J. M., Kemeny, D. M., et al. (2009). LysRS serves as a key signaling molecule in the immune response by regulating gene expression. Mol. Cell 34, 603-611. doi: 10.1016/j.molcel.2009.05.019

Yudin, D., and Fainzilber, M. (2009). Ran on tracks-cytoplasmic roles for a nuclear regulator. J. Cell Sci. 122, 587-593. doi: 10.1242/jcs.015289

Zhang, C., and Kuspa, A. (2009). Transcriptional down-regulation and rRNA cleavage in Dictyostelium discoideum mitochondria during Legionella pneumophila infection. PLoS ONE 4:e5706. doi: 10.1371/journal.pone.0005706
Conflict of Interest Statement: The authors declare that the research was conducted in the absence of any commercial or financial relationships that could be construed as a potential conflict of interest.

Copyright (c) 2018 Swart, Harrison, Eichinger, Steinert and Hilbi. This is an openaccess article distributed under the terms of the Creative Commons Attribution License (CC BY). The use, distribution or reproduction in other forums is permitted, provided the original author(s) and the copyright owner are credited and that the original publication in this journal is cited, in accordance with accepted academic practice. No use, distribution or reproduction is permitted which does not comply with these terms. 